\title{
Extension of smooth functions in infinite dimensions II: manifolds
}

\author{
by \\ C. J. AtKin (Wellington)
}

\begin{abstract}
Let $M$ be a separable $\mathrm{C}^{\infty}$ Finsler manifold of infinite dimension. Then it is proved, amongst other results, that under suitable conditions of local extensibility the germ of a $\mathrm{C}^{\infty}$ function, or of a $\mathrm{C}^{\infty}$ section of a vector bundle, on the union of a closed submanifold and a closed locally compact set in $M$, extends to a $\mathrm{C}^{\infty}$ function on the whole of $M$.
\end{abstract}

1. Introduction. In the preceding paper [2], I presented a theorem, $[2$, 5.1], which asserts the possibility of extending functions of various classes from a finite union of convex sets to the whole of a locally convex space. A particular example, assuming the space to be normed, would be the Fréchet $\mathrm{C}^{\infty}$ differentiability class. The purpose of this paper is to show how that theorem may be used to obtain extension theorems for functions of a given class from suitable subsets of a manifold to the whole manifold.

An extension theorem will say that any "function" of a certain class defined on a subset $X$ of a space $M$ is necessarily the "restriction" of a "function" of some related class on $M$. The "functions" need not always be functions in the usual sense, and "restriction" may not be a completely obvious procedure, as with the trace of a Sobolev function on the boundary of its domain. Setting aside such possibilities, there are several kinds of extension theorem that one might consider. One might ask simply for a function on $M$ whose restriction to $X$ is given; or one might, for instance, also ask that its infinitesimal behaviour at points of $X$ should be prescribed. This is the content of Whitney's famous theorem, [11], which in effect extends suitable $\mathrm{C}^{\infty}$-jets on a closed set $X$ in $\mathbb{R}^{n}$ to $\mathrm{C}^{\infty}$ functions on $\mathbb{R}^{n}$. One might also ask for a function on $M$ whose germ at $X$ agrees with a given germ, that is, it agrees on some unspecified small neighbourhood of $X$ with a given function on some neighbourhood of $X$. The principal theorem presented here, 5.5, is of this last kind: if $X$ is a suitable subset (a closed locally compact subset, a closed submanifold, or a union of both) of the manifold $M$,

2000 Mathematics Subject Classification: Primary 46T20. 
a "smooth" function $f$ defined on a neighbourhood $V$ of $X$ coincides on a certain smaller neighbourhood $W$ of $X$ with a "smooth" function on $M$. The neighbourhood $W$ depends only on $V$ and $X$.

When $X$ is just a pair of points, 5.5 has the following very weak but interesting consequence, proved as 5.6 below:

Lemma 1.1. Let $M$ be a paracompact $\mathrm{C}^{\infty}$ manifold modelled on the $B a$ nach space $C$ or on the Banach space $\ell^{1}$. The class of real-valued $\mathrm{C}^{\infty}$ functions on $M$ separates points.

This answers the question raised by Elworthy in [5], and mentioned in the introduction to [2]. In fact, the restriction to $C$ and $\ell^{1}$ as models is quite unnecessary, in view of extension properties and smoothing theorems that will be presented in later papers.

For the sake of general applicability, my principal results are presented in terms of the extension of sections of fibrations rather than of functions. There are theorems similar to 5.5 that may be derived from $[2,5.5]$; I have omitted them, since their proofs are essentially the same, and their hypotheses inevitably more complicated. Some of the definitions of $[2, \S 2]$ will be assumed.

There are two obvious difficulties in applying the results of [2] on manifolds. [2, 5.1] and its variants depend on convexity (see [2, 3.3] for some remarks on this) and on "extensibility" of the functions considered (see $[2$, 1.5] for the definition). One cannot usually expect either of these properties to be preserved by the coordinate transformations of a manifold. However, for a $\mathrm{C}^{1}$ manifold modelled on a separable normed space with the Fréchet definition of differentiability, the charts may be so chosen that the coordinate transformations preserve convexity "approximately", and this is enough for the proof here. The requirement that the functions in question be extensible is not so simply overcome, but, as I pointed out at $[2,1.3]$, there are many standard models for which the $\mathrm{C}^{\infty}$ class, at least, consists entirely of extensible functions. As I have already hinted, I hope to demonstrate elsewhere that, for many cases of interest, the requirement of extensibility either is satisfied automatically or can be satisfied by a careful choice of charts.

When the model is not a normed space, there is no natural notion of differentiability, and the definitions usually presented (see [7] and [8], for example) are insufficient to support the "approximate convexity" arguments used in the normed case. Thus the result that I present for such models, Theorem 6.1, is much weaker, but not altogether uninteresting.

Whitney's method of proving his extension theorem cannot be applied in infinite dimensions, since it employs smooth partitions of unity, which need not always exist ([3], [4]), and covers by bounded cubes with disjoint interiors, which never can. There are, in fact, well known counterexamples to 
many natural conjectures. If $f$ is a function which is 1 on a neighbourhood of the origin of a separable normed space $E$ of infinite dimension and is 0 on a neighbourhood of the unit sphere $S$ of $E$, any $\mathrm{C}^{\infty}$ extension of $f$ to the whole of $E$ may easily be adjusted to give a non-zero $\mathrm{C}^{\infty}$ function of bounded support, which, by [3] or [4], is impossible in many spaces (such as $C$ and $\left.\ell^{1}\right)$.

At first glance, the reason for this counterexample is that $S$ separates $E$. However, the set $S \times\{0\}$ in $E \times E$ is homotopy-negligible (the inclusion

$$
(E \times E) \backslash(S \times\{0\}) \rightarrow E \times E
$$

is a homotopy equivalence), and yet any $\mathrm{C}^{\infty}$ extension to $E \times E$ of a function which is 1 at $(0,0)$ and 0 on $S \times\{0\}$ would also lead to a non-zero $\mathrm{C}^{\infty}$ function of bounded support in $E \times\{0\}$. In these examples, it is immaterial that $S$ is a norm-sphere; any bounded closed set separating a subspace of $E$ that does not admit $\mathrm{C}^{\infty}$ non-zero functions of bounded support would give a like result. It seems, then, that certain sets in some spaces are "bad" for extension problems, and that their "badness" is a rather subtle property, related not merely to their topological embedding in the ambient space but also to its analytical or geometrical character. There is a vague analogy with the theory of several complex variables, in which spheres are similarly "bad" for holomorphic functions. One cannot expect a simple characterization of "good" sets for a given extension problem, since it would have to depend on the spaces and the function classes involved; nevertheless, the theorems of this paper do assert the "goodness", for the problems considered, of certain rather simple kinds of set.

The arguments here were developed over a very long period. I am grateful to the Institutes of Mathematics of the University of Warsaw and of the Polish Academy of Sciences for the hospitality which enabled me at last to put them into a reasonable form, and to the referee for helpful suggestions.

\section{Localization and fibrations}

Definition 2.1. Suppose that $\mathcal{C}$ is a category of mappings between topological spaces. (There may be very few objects in the category $\mathcal{C}$.) Extend $\mathcal{C}$ by "localization" to the class $\mathcal{L C}$ of locally- $\mathcal{C}$ mappings between open sets of objects of $\mathcal{C}$, as follows.

Let $U$ be an open set in $X$ and $V$ an open set in $Y$, where both $X$ and $Y$ are objects of $\mathcal{C}$, and let $i: V \rightarrow Y$ denote the inclusion. Then $f: U \rightarrow V$ belongs to $\mathcal{L C}$ if and only if, for each $x \in U$, there exist a neighbourhood $W$ of $x$ in $U$ and a morphism $g: X \rightarrow Y$ of $\mathcal{C}$ such that $g|W=i \circ f| W$.

In the language of $[2,1.5], \mathcal{L C}$ consists of the $\mathcal{C}$-extensible mappings of open sets of objects of $\mathcal{C}$. If $\mathcal{C}$ consists of continuous mappings, then so does 
$\mathcal{L C}$, which is itself a category. The mappings in $\mathcal{L C}$ are automatically locally defined (see $[2,1.4]$ ).

Definition 2.2. The category $\mathcal{C}$ is admissible if its objects are topological vector spaces and, for each pair $E, F$ of objects of $\mathcal{C}$, the $\mathcal{C}$-morphisms from $E$ to $F$ form an $F$-admissible class (see $[2,1.7]$ ).

It then follows that, if $X$ is open in $E$ and $Y$ is open and convex in $F$, the $\mathcal{L C}$-morphisms $X \rightarrow Y$ are $Y$-admissible in the sense of $[2,1.7]$.

Definition 2.3. Given a category $\mathcal{C}$, as in 2.1 above, which consists of continuous maps, an $\mathcal{L C}$-manifold is a manifold, modelled on an object $E$ of $\mathcal{C}$, for which all the coordinate transformations are isomorphisms in $\mathcal{L C}$.

In other words, $\mathcal{L C}$ determines a pseudogroup $\mathfrak{G}_{E}(\mathcal{C})$ of transformations in $E$, consisting of the mappings $f: U \rightarrow V$ between open subsets of $E$ which are in $\mathcal{L C}$ and have inverses $f^{-1}: V \rightarrow U$ also in $\mathcal{L C}$. An $\mathcal{L C}$-manifold modelled on $E$ is then a $\mathfrak{G}_{E}(\mathcal{C})$-manifold. I shall always write of $\mathcal{L C}$-manifolds and $\mathcal{L C}$-charts, rather than mention the pseudogroup $\mathfrak{G}_{E}(\mathcal{C})$; this accords with familiar usage.

The category $\mathcal{L C}$ may now be further extended. If $M$ and $N$ are $\mathcal{L C}$ manifolds, modelled respectively on the objects $E$ and $F$ of $\mathcal{C}$, a mapping $f: M \rightarrow N$ is described as being in $\mathcal{L C}$, or as being an $\mathcal{L C}$-mapping, if, for each $x \in M$, there are open neighbourhoods $U$ of $x$ in $M$ and $V$ of $f(x)$ in $N$, and $\mathcal{L C}$-manifold charts $\phi: U \rightarrow \phi(U) \subseteq E, \psi: V \rightarrow \psi(V) \subseteq F$, where $\phi(U)$ is open in $E$ and $\psi(V)$ is open in $F$, such that $f(U) \subseteq V$ and $\psi \circ(f \mid U) \circ \phi^{-1}: \phi(U) \rightarrow \psi(V)$ is in $\mathcal{L C}$.

If this condition holds for one specific choice of $\mathcal{L C}$-manifold charts $\phi$ and $\psi$ with the given domains $U$ and $V$, it also holds for all other choices. Since $\mathcal{C}$ is assumed to consist of continuous maps, an $\mathcal{L C}$-mapping of $\mathcal{L C}$ manifolds must be continuous; and such mappings form a category, which may be called the $\mathcal{L C}$-category. (This name is somewhat ambiguous, in view of 2.1, but it is an ambiguity of a kind familiar from the $\mathrm{C}^{p}$ category.)

An open subset $U$ of an $\mathcal{L C}$-manifold $M$ itself clearly has a unique $\mathcal{L C}$ manifold structure such that the inclusion $U \rightarrow M$ is an $\mathcal{L C}$-mapping. Furthermore, any $\mathcal{L C}$-mapping into $M$ whose image is included in $U$ is then an $\mathcal{L C}$-mapping into $U$.

Definition 2.4. A category $\mathcal{D}$ is applicable if its objects are open sets in topological vector spaces, its morphisms are continuous mappings, and, together with any morphism $\phi$, it contains all mappings obtained from $\phi$ by restriction to an open subset of its domain or image. (The categories $\mathcal{L C}$ are examples of applicable categories.)

Definition 2.5. Suppose that the topological vector space $E$ is an object of the applicable category $\mathcal{D}$, and $F$ is a linear subspace of $E$. Let $M$ be 
a $\mathcal{D}$-manifold modelled on $E$. A $\mathcal{D}$-submanifold of $M$ modelled on $F$ is a nonnull subset $N$ of $M$ which may be covered by $\mathcal{D}$-charts $\phi: U \rightarrow \phi(U) \subseteq E$ of $M$ such that $\phi(U \cap N)=\phi(U) \cap F$. Such charts may be called $\mathcal{D}$ submanifold charts for $N$.

When $E$ is a normed space and $M$ is a $\mathrm{C}^{1}$-manifold modelled on $E$, a $\mathrm{C}^{1}$-submanifold modelled on $F$ is, similarly, a non-null subset $N$ of $M$ such that every point of $M$ is contained in the domain of some $\mathrm{C}^{1}$ chart of $M$, $\phi: U \rightarrow \phi(U) \subseteq E$, for which $\phi(U \cap N)=\phi(U) \cap F$.

The submanifolds just defined are the only ones that will be considered in this paper, although in other contexts other definitions are appropriate. In this case, the submanifold $N$ is closed in $M$ if and only if its model $F$ is closed in $E$.

REMARK 2.6. The $\mathcal{L C}$-category is merely an ad hoc tool for the convenient formulation of theorems, and is not a useful general setting for geometry, even when $\mathcal{C}$ is the $\mathrm{C}^{\infty}$ category; for it is not, in principle, closed under simple analytical operations such as the solution of ordinary differential equations. Of course, if one restricts attention to many "standard" normed spaces, $\mathcal{L} \mathrm{C}^{\infty}=\mathrm{C}^{\infty}$ (see $\left.[2,1.3]\right)$, and there is no problem.

As defined here, an $\mathcal{L C}$-submanifold need not even be an $\mathcal{L C}$-manifold in a natural way, although a $\mathrm{C}^{1}$-submanifold of a $\mathrm{C}^{1}$-manifold does have a natural $\mathrm{C}^{1}$ structure and the same is true for many other common choices of $\mathcal{C}$.

Definition 2.7. Given the category $\mathcal{C}$ as in 2.1 and an object $E$ of $\mathcal{C}$, a subspace $F$ of $E$ is $\mathcal{C}$-imbedded if the inclusion $i: F \rightarrow E$ belongs to $\mathcal{C}$ and any mapping $g: H \rightarrow F$ (where $H$ is an object of $\mathcal{C}$ ) such that $i \circ g \in \mathcal{C}$ itself belongs to $\mathcal{C}$.

LEMMA 2.8. Let $N$ be an $\mathcal{L C}$-submanifold, modelled on the subspace $F$, of the $\mathcal{L C}$-manifold $M$ modelled on the space $E$, and suppose that $F$ is $\mathcal{C}$-imbedded in $E$. Then there is a unique $\mathcal{L C}$-structure on $N$ such that the inclusion $N \rightarrow M$ is an $\mathcal{L C}$-mapping.

Proof. If $\phi: U \rightarrow \phi(U) \subseteq E$ and $\psi: V \rightarrow \psi(V) \subseteq E$ are $\mathcal{L C}$-charts of $M$ such that $\phi(U \cap N)=\phi(U) \cap F$ and $\psi(V \cap N)=\psi(V) \cap F$, then the coordinate transformation

$$
\chi:=(\phi \mid U \cap V) \circ(\psi \mid U \cap V)^{-1}: \psi(U \cap V) \rightarrow \phi(U \cap V)
$$

in $\mathcal{L C}$ carries $\psi(U \cap V) \cap F$ into $\phi(U \cap V) \cap F$. However, if $F$ is $\mathcal{C}$-imbedded in $E$, this implies that $\chi \mid \psi(U \cap V) \cap F$ as a mapping into $\phi(U \cap V) \cap F$ is itself in $\mathcal{L C}$. The $\mathcal{L C}$-structure on $N$ thus defined is the only possible one making the inclusion $\mathcal{L C}$. 
Definition 2.9. Let $E, F$ be objects of $\mathcal{C}$; suppose that, whenever mappings $\phi: E \rightarrow E$ and $\psi: F \rightarrow F$ are in $\mathcal{C}, \phi \times \psi: E \times F \rightarrow E \times F$ is also in $\mathcal{C}$. Then, for any two $\mathcal{L C}$-manifolds $M, N$ with models $E, F$ respectively, there is an $\mathcal{L C}$-manifold $M \times N$ defined by an atlas whose charts are the products of charts for $M$ and for $N$ in the usual way.

Assume further that the projections $E \times F \rightarrow E$ and $E \times F \rightarrow F$, and the diagonal map $E \rightarrow E \times E$, are in $\mathcal{C}$ (compare 2.8); then both the coordinate projections $p_{1}: M \times N \rightarrow M$ and $p_{2}: M \times N \rightarrow N$, and the diagonal map $\nabla: M \rightarrow M \times M$, are also in $\mathcal{C}$.

By a locally trivial $\mathcal{L C}$-fibration (with base $M$, whose model is $E$, and fibre $N$, whose model is $F$ ) I mean an $\mathcal{L C}$-mapping $\pi: \mathcal{E} \rightarrow M$ of $\mathcal{L} \mathcal{C}$ manifolds, such that, for each $x \in M$, there are an open neighbourhood $U$ of $x$ in $M$ (hence $U$ and $\pi^{-1}(U)$ have natural $\mathcal{L C}$-structures, by the remarks after 2.3) and an $\mathcal{L C}$-isomorphism $\phi: \pi^{-1}(U) \rightarrow U \times N$ for which $p_{1} \circ \phi=\pi$. Such a neighbourhood $U$ is called an $\mathcal{L C}$-trivializing neighbourhood (of $x$ in $M$, for the fibration $\pi$ ), and such an $\mathcal{L C}$-diffeomorphism $\phi$ may be called an $\mathcal{L C}$-local trivialization for $\pi$ over the base $U$. Any smaller open neighbourhood of $x$ will then also be $\mathcal{L C}$-trivializing, an $\mathcal{L C}$-local trivialization being furnished by the restriction of $\phi$. The notion of the restriction of the locally trivial $\mathcal{L} \mathcal{C}$-fibration $\pi$, also called $\mathcal{E}$, to a (suitable -for instance open) subset $V$ of $M$ is defined as usual, and will be denoted by $\mathcal{E} \mid V$.

By an $\mathcal{L C}$-vector fibration (with base $M$ and fibre $E$ ) I mean a locally trivial $\mathcal{L C}$-fibration $\pi: \mathcal{E} \rightarrow M$ for which each fibre is given the structure of a topological vector space isomorphic to $E$, in such a way that $M$ may be covered by the bases $U$ of $\mathcal{L C}$-local trivializations $\phi: \pi^{-1}(U) \rightarrow U \times E$ whose restriction to each fibre is a topological linear isomorphism. These may be called $\mathcal{L C}$-local vector trivializations of $\pi$.

Equivalently, the transformation $\psi \circ \phi^{-1}$ between two such local trivializations is to be a topological linear isomorphism in each fibre. This is a weaker definition than is usually given (see [9]) for a vector bundle with normed infinite-dimensional fibres. For a $\mathrm{C}^{0}$-vector bundle whose fibre is a normed space $E$, the bundle transition functions $\theta_{U V}: U \cap V \rightarrow \mathrm{GL}(E)$ between two local bundle trivializations $\phi: \pi^{-1}(U) \rightarrow U \times E$ and $\psi: \pi^{-1}(V) \rightarrow V \times E$ are defined by setting $\theta_{U V}(x) \xi:=\psi \circ \phi^{-1}(x, \xi)$ for any $x \in U \cap V$ and $\xi \in E$, and are required to be continuous as maps into $\operatorname{GL}(E)$ with the uniform norm topology. This condition is evidently needed if the notion of a Finsler structure in the bundle is to make sense. (For clarity, I shall call such a Finsler structure a bundle Finsler structure; a Finsler structure on a manifold, which is the same as a bundle Finsler structure in its tangent bundle, may be called a tangential Finsler structure.) $\mathrm{A} \mathrm{C}^{1}$-vector fibration is a $\mathrm{C}^{0}$-vector bundle, since the transition functions may be obtained by 
differentiation at the points of the zero-section; but it is also a $\mathrm{C}^{1}$-manifold, which a $\mathrm{C}^{0}$-vector bundle need not be. This is the "loss of differentiability problem" discussed in [5].

Definition 2.10. Let $\pi: \mathcal{E} \rightarrow M$ be an $\mathcal{L C}$-vector fibration with base $M$ and fibre $E$, and suppose that $\mathcal{E}_{1}$ is a subset of $\mathcal{E}$ and $G$ is an $\mathcal{C}$-imbedded vector subspace of $E$. Then $\pi_{1}:=\pi \mid \mathcal{E}_{1}$ is an $\mathcal{L C}$-vector subfibration of $\pi$, with fibre $G$, if $M$ may be covered by the bases $U$ of $\mathcal{L C}$-local trivializations $\phi: \pi^{-1}(U) \rightarrow U \times E$ such that

$$
\phi\left(\pi^{-1}(U) \cap \mathcal{E}_{1}\right)=U \times G .
$$

In such a case, $\pi_{1}$ is an $\mathcal{L C}$-vector fibration in its own right (as $G$ is $\mathcal{C}$ imbedded in $E$ ).

Provided that the category $\mathcal{C}$ satisfies suitable conditions on the formation of quotients, one may construct a quotient $\mathcal{L C}$-vector fibration $\pi / \pi_{1}$ (or $\mathcal{E} / \mathcal{E}_{1}$ ), which is an $\mathcal{L C}$-vector fibration with base $M$ and fibre $E / G$. (The category $\mathrm{C}^{0}$ is certainly satisfactory for this purpose.) Similarly, an $\mathcal{L C}$-morphism of $\mathcal{L C}$-vector fibrations is an $\mathcal{L C}$-map which respects fibres and is linear in each fibre; the inclusion map of an $\mathcal{L C}$-vector subfibration, and the quotient map when it is defined, are $\mathcal{L C}$-morphisms. $\mathcal{L C}$-morphisms may be added and multiplied by scalars. There are similar definitions for $\mathrm{C}^{0}$-vector bundles.

Definition 2.11. Let $\pi: L \rightarrow M$ be a locally trivial $\mathcal{L C}$-fibration, as above. A section of $\pi$ over a subset $X$ of $M$ is of course a function $\sigma: X \rightarrow L$ such that $\pi \circ \sigma$ is the inclusion of $X$ in $M$. When $U$ is an open subset of $M$, an $\mathcal{L C}$-section of $\pi$ over $U$ is a section which is an $\mathcal{L C}$-mapping.

An $\mathcal{L C}$-local trivialization $\phi: \pi^{-1}(U) \rightarrow U \times N$ establishes a one-one correspondence between $\mathcal{L C}$-sections of $\pi$ over $U$ and $\mathcal{L C}$-mappings $U \rightarrow N$; the section $\sigma$ defines the mapping $p_{2} \circ \phi \circ \sigma$, and the mapping $f$ gives the section $\sigma$, where $\sigma(x):=\phi^{-1} \circ(1 \times f) \circ \nabla(x)$.

REMARK 2.12. The usual constructions, such as pullbacks, may be carried out for locally trivial $\mathcal{L C}$-fibrations, provided that $\mathcal{C}$ is large enough for the constructions to make sense. Therefore, at 3.6, I shall restrict discussion to $\mathcal{L C}$-sections of a locally trivial $\mathcal{L C}$-fibration $\pi: L \rightarrow M$. There are similar results for $\mathcal{L C}$-liftings to $L$ of an $\mathcal{L C}$-mapping $k: R \rightarrow M$, where $R$ is another $\mathcal{L C}$-manifold; they may be proved either by the same method, with some slight complications of notation, or, if $\mathcal{C}$ is a suitable category, by associating such liftings with sections of $k^{*} \pi$.

3. An extension theorem. This section is devoted to the proof of the technical result 3.6, whose application occupies the rest of the paper. The proof is inductive; its structure is very similar to the proof of $[2,4.2]$, and 
it depends on $[2,5.1]$. In particular, the definitions of $[2, \S 2]$ will be used without explanation. $\mathcal{D}$ is to denote an applicable category (see 2.4), and the convex envelope of a subset $Q$ in a vector space $E$ will be denoted by $\operatorname{co}(Q)$.

Definition 3.1. Let $M$ be a $\mathcal{D}$-manifold. An extension family in $M$ is a pair $\left(\left\{\left(U^{j}, U_{0}^{j}\right): j \in J\right\}, \Theta\right)$ consisting of a class of pairs of open sets indexed by a finite or countable set $J$, and of a subset $\Theta$ of $J$, which satisfies the conditions:

(i) the class $\left\{U_{0}^{j}: j \in J\right\}$ covers $M$;

(ii) for any $\psi \in J \backslash \Theta$, the set $\Theta_{\psi}:=\left\{\theta \in \Theta: U^{\psi} \cap U^{\theta} \neq \emptyset\right\}$ is finite;

(iii) for any $j \in J, \operatorname{cl}_{M}\left(U_{0}^{j}\right) \subseteq U^{j}$.

For any extension family, I shall write

$$
U_{0}(\Theta):=\bigcup_{i \in \Theta} U_{0}^{i} \text { and } U(\Theta):=\bigcup_{i \in \Theta} U^{i} .
$$

Lemma 3.2. If $\left(\left\{\left(U^{j}, U_{0}^{j}\right): j \in J\right\}, \Theta\right)$ is an extension family in $M$, then $\operatorname{cl}_{M}\left(U_{0}(\Theta)\right) \subseteq U(\Theta)$.

Proof. Suppose $x \in \operatorname{cl}_{M}\left(U_{0}(\Theta)\right) \backslash U_{0}(\Theta)$. By 3.1(i), there is some $\psi \in$ $J \backslash \Theta$ such that $x \in U_{0}^{\psi}$, and $U^{\psi}$ meets $U_{0}^{\theta}$ (for $\theta \in \Theta$ ) only when $\theta$ is in the finite set $\Theta_{\psi}$. Hence, $x \in \operatorname{cl}_{M}\left(\bigcup_{\theta \in \Theta_{\psi}} U_{0}^{\theta}\right)=\bigcup_{\theta \in \Theta_{\psi}} \operatorname{cl}_{M}\left(U_{0}^{\theta}\right)$, and $\operatorname{cl}_{M}\left(U_{0}^{\theta}\right) \subseteq U^{\theta} \subseteq U(\Theta)$ for $\theta \in \Theta$.

Definition 3.3. If $M$ is a $\mathcal{D}$-manifold and $\mathfrak{V}$ is an open cover of $M$, a $\mathfrak{V}$-extension system in $M$ is an extension family $\left(\left\{\left(U^{j}, U_{0}^{j}\right): j \in J\right\}, \Theta\right)$ satisfying the additional condition

(iv) for any finite subset $T \subseteq J$ and any $\psi \in J \backslash \Theta$, there is a $\mathcal{D}$-chart $\omega_{\psi, T}: O_{\psi, T} \rightarrow E$ for $M$ such that

(a) $O_{\psi, T} \subseteq V$ for some $V \in \mathfrak{V}$,

(b) when $\bar{k}, l \in T$ and $U^{\psi} \cap U^{k} \neq \emptyset \neq U^{k} \cap U^{l}$, then $U^{l} \subseteq O_{\psi, T}$ and $\operatorname{co}\left(\omega_{\psi, T}\left(U_{0}^{l}\right)\right) \prec \omega_{\psi, T}\left(U^{l}\right)$.

If $\mathfrak{V}$ consists of all the open sets of $M$, one might speak of an extension system.

If one takes $T:=\{j\}$, (iv)(b) shows that $\left\{U^{j}\right\}$ must refine $\mathfrak{V}$.

REMARK 3.4. In most respects, the above Definition 3.3 is what is needed in the proof of 3.6. However, the full strength of (iv) is superfluous, for $J$ is taken in 3.6 to be a subset of $\mathbb{N}$ and, for a given $\psi \in J \backslash \Theta$, the only set $T$ that is used is $(J \cap[1, \psi]) \cup\left(\bigcup_{j \in J \cap[1, \psi]} \Theta_{j}\right)$ (so that the chart $\omega_{\psi, T}$ depends only on $\psi$ ). Definition 3.3 could, therefore, be weakened somewhat, but at the cost of assuming a specific ordering of $J$. 
The details of the argument are somewhat arbitrary, and, in fact, to apply 3.6, I shall prove the substantially stronger properties 3.5. As a definition, therefore, 3.3 is an ugly compromise; but it has the potential advantage that, as in the suggested weaker version, (iv)(b) only imposes finitely many conditions on $\omega_{\psi, T}$.

The definitions here do not require an $\mathcal{L C}$-manifold, and it is in principle quite permissible, albeit not usually possible, that $\bigcup_{\psi \in J \backslash \Theta} U_{0}^{\psi}$ itself should be the whole of $M$.

If 3.1(ii) were weakened to require only that $\left\{\theta \in \Theta: U^{\psi} \cap U_{0}^{\theta} \neq \emptyset\right\}$ be finite, with a corresponding change in 3.3(iv)(b), the later arguments could with some effort be improved to show that any closed set in a Finsler manifold is an $\mathcal{L C}$-extension core in the sense of 3.9. That conclusion is false, as I noted in $\S 1$; the induction in 3.6 would indeed fail. But it is curious that the form of (ii) is so crucial for the extension theorems.

Definition 3.5. Again, let $M$ be a $\mathcal{D}$-manifold and $\mathfrak{V}$ an open cover of $M$. A strong $\mathfrak{V}$-extension system in $M$ is a pair $\left(\left\{\left(U^{j}, U_{0}^{j}\right): j \in J\right\}, \Theta\right)$ satisfying 3.1(i)-(iii) and

(iv') for any $\psi \in J \backslash \Theta$, there is a $\mathcal{D}$-chart $\omega_{\psi}: O_{\psi} \rightarrow E$ for $M$ such that

(a) there is some $V \in \mathfrak{V}$ such that $O_{\psi} \subseteq V$,

(b) when $k, l \in J$ and $U^{\psi} \cap U^{k} \neq \emptyset \neq \bar{U}^{k} \cap U^{l}$, then $U^{l} \subseteq O_{\psi}$ and $\operatorname{co}\left(\omega_{\psi}\left(U_{0}^{l}\right)\right) \prec \omega_{\psi}\left(U^{l}\right)$.

Proposition 3.6. Suppose $\mathcal{C}$ is an admissible category (see 2.2). Suppose that $\pi: L \rightarrow M$ is a locally trivial $\mathcal{L C}$-fibration of $\mathcal{L C}$-manifolds, with base $M$ and fibre $N$ (see 2.9), where $N$ is $\mathcal{L C}$-diffeomorphic to the open convex subset $Y$ of the topological vector space $F$ and $M$ has the locally convex Lindelöf model E.

Let $\mathfrak{V}$ be any cover of $M$ by open sets $V$ in $M$ for which there is an $\mathcal{L C}$-local trivialization $\phi: \pi^{-1}(V) \rightarrow V \times N$ of $\pi$. Suppose that the pair $\left(\left\{\left(U^{j}, U_{0}^{j}\right): j \in J\right\}, \Theta\right)$ is a $\mathfrak{V}$-extension system in $M$. Then, for any $\mathcal{L C}$ section $\tau$ of $\pi$ over $U(\Theta)$, there exists an $\mathcal{L C}$-section $\sigma$ of $\pi$ over $M$ such that $\sigma\left|U_{0}(\Theta)=\tau\right| U_{0}(\Theta)$.

Proof. (a) Taking $T:=(J \cap[1, \psi]) \cup\left(\bigcup_{j \in J \cap[1, \psi]} \Theta_{j}\right)$ in 3.3(iv), one finds that, for the given $\mathfrak{V}$ and for each $\psi \in J \backslash \Theta$, there exist both an $\mathcal{L C}$-chart $\omega_{\psi}: O_{\psi} \rightarrow E$ (I write $\omega_{\psi}$ for $\omega_{\psi, T}$ and $O_{\psi}$ for $O_{\psi, T}$ ) with the stated properties, and an $\mathcal{L C}$-local trivialization $\phi_{\psi}: \pi^{-1}\left(O_{\psi}\right) \rightarrow O_{\psi} \times Y$, where the $\mathcal{L C}$-diffeomorphism of the fibre with $Y$ has been assimilated into $\phi_{\psi}$.

(b) Define, for $r=1,2, \ldots$,

$$
\begin{aligned}
& T_{r}:=\{i:(1 \leq i \leq r) \text { or } \\
&\left.\left(i \in \Theta \&(\exists \psi \notin \Theta)\left(1 \leq \psi \leq r \& U^{\psi} \cap U^{i} \neq \emptyset\right)\right)\right\} .
\end{aligned}
$$


Clearly $\{1, \ldots, r\} \subseteq T_{r} \subseteq T_{r+1}$ for each $r \geq 1$. Furthermore, hypothesis 3.1(ii) implies that $T_{r}$ must be finite. Let $\mathcal{A}(r):=\left\{U^{i}: i \in T_{r}\right\}$ and $\mathcal{B}(r):=\left\{U_{0}^{i}: i \in T_{r}\right\}$, and recall the terminology of $[2,2.10]$. The inductive hypotheses at stage $r$ are as follows:

$\mathcal{H}_{1}(r)$ : for every simplex $\Delta$ of $\mathcal{A}(r)$, there is an $\mathcal{L C}$-section $\sigma_{r}(\Delta)$ of $\pi$ over $A(r)^{\Delta}$, the star $\bigcup_{i \in \Delta} U^{i}$ of the simplex $\Delta$ in $\mathcal{A}(r)$;

$\mathcal{H}_{2}(r)$ : whenever $\Delta_{1}, \Delta_{2}$ are simplices of $\mathcal{A}(r)$ and $i \in \Delta_{1} \cap \Delta_{2}$,

$$
\sigma_{r}\left(\Delta_{1}\right)\left|U_{0}^{i}=\sigma_{r}\left(\Delta_{2}\right)\right| U_{0}^{i}
$$

(The $\mathcal{L C}$-sections $\sigma_{r}(\Delta)$ will therefore fit together to define an $\mathcal{L C}$-section $\sigma_{r}$ over the carrier $B(r):=\bigcup_{i \in T_{r}} U_{0}^{i}$.)

$\mathcal{H}_{3}(r)$ : for each simplex $\Delta$ of $\mathcal{A}(r)$, and any $i \in \Delta \cap \Theta$,

$$
\sigma_{r}(\Delta)\left|U_{0}^{i}=\tau\right| U_{0}^{i}
$$

(In other words, $\sigma_{r}\left|U_{0}^{i}=\tau\right| U_{0}^{i}$ for each $i \in T_{r} \cap \Theta$ ).

(c) The induction begins with $r=0, T_{0}=\emptyset, \mathcal{A}(0)=\mathcal{B}(0)=\emptyset$.

(d) Suppose the inductive hypotheses satisfied for some $r \geq 0$. If $r+1 \in T_{r}$, then $r+1 \in \Theta$ and $T_{r+1}=T_{r}$. If $r+1 \notin T_{r}$, then $T_{r+1} \backslash T_{r}$ consists of $r+1$ and, if $r+1 \notin \Theta$, also of those $\theta \in \Theta$ for which $U^{\theta}$ meets $U^{r+1}$ but no earlier $U^{\psi}$ with $\psi \notin \Theta$ (that is, $\left.\theta \in \Theta_{r+1} \backslash\left(\bigcup_{r \geq \psi \notin \Theta} \Theta_{\psi}\right)\right)$. Thus the simplices of $\mathcal{A}(r+1)$ fall, by (1), into three disjoint classes, which will be treated separately:

I. simplices of $\mathcal{A}(r)$;

II. simplices not in $\mathcal{A}(r)$ of which $r+1$ is not a vertex;

III. simplices not in $\mathcal{A}(r)$ with $r+1$ as a vertex.

(Simplices of classes II and III only exist when $r+1 \notin T_{r}$.)

(e) If $\Delta$ is a simplex of $\mathcal{A}(r+1)$ in class I, define $\sigma_{r+1}(\Delta):=\sigma_{r}(\Delta)$.

(f) Next, suppose that $\Delta$ is a simplex of $\mathcal{A}(r+1)$ in class II. As $\Delta$ is not a simplex of $\mathcal{A}(r)$, it has at least one vertex $k>r+1$, necessarily in $\Theta$, such that $U^{k}$ does not meet any $U^{\psi}$ for which $1 \leq \psi \leq r$ and $\psi \notin \Theta$. But this implies that such vertices $\psi$ cannot belong to $\Delta$ at all, so that $\Delta \subseteq \Theta$. Hence, $A(r+1)^{\Delta} \subseteq U(\Theta)$, and one may define $\sigma_{r+1}(\Delta):=\tau \mid A(r+1)^{\bar{\Delta}}$.

(g) The inductive hypotheses $\mathcal{H}_{1}(r+1), \mathcal{H}_{2}(r+1)$, and $\mathcal{H}_{3}(r+1)$ are satisfied when $\Delta_{1}, \Delta_{2}, \Delta$ are simplices in class I, because, by (e), they reduce to $\mathcal{H}_{1}(r), \mathcal{H}_{2}(r)$, and $\mathcal{H}_{3}(r)$. When $\Delta$ is in class II, the definition (f) instantly gives $\mathcal{H}_{3}(r+1)$. Ergo, $\mathcal{H}_{2}(r+1)$ follows from $\mathcal{H}_{3}(r+1)$ and $\mathcal{H}_{3}(r)$ when $\Delta_{1}$ is in class II and $\Delta_{2}$ is in class I or class II, since the vertices of $\Delta_{1}$ are all in $\Theta$ anyway.

(h) The non-trivial case is that of a simplex of class III. Recall that, for such a simplex to exist, $r+1 \notin T_{r}$, and any vertex $j>r+1$ must belong 
to $\Theta_{r+1}$. Let

$$
H_{r}:=\left\{k \in T_{r+1}:\left(\exists j \in T_{r+1}\right)\left(U^{r+1} \cap U^{j} \neq \emptyset \& U^{k} \cap U^{j} \neq \emptyset\right)\right\} .
$$

Take the $\mathcal{L C}$-chart $\omega_{r+1}: O_{r+1} \rightarrow E$ and the $\mathcal{L C}$-local trivialization of $\pi$ over $O_{r+1}, \phi_{r+1}: \pi^{-1}\left(O_{r+1}\right) \rightarrow O_{r+1} \times Y$, as before. Thus, for every $k \in H_{r}$,

$$
U^{k} \subseteq O_{r+1} \quad \text { and } \quad \operatorname{co}\left(\omega_{r+1}\left(U_{0}^{k}\right)\right) \prec \omega_{r+1}\left(U^{k}\right) \text {. }
$$

Choose a convex open neighbourhood $W$ of 0 in $E$ such that, for all $k \in H_{r}$,

$$
R_{k, r}:=\operatorname{co}\left(\omega_{r+1}\left(U_{0}^{k}\right)\right)+W \prec \omega_{r+1}\left(U^{k}\right) .
$$

$R_{k, r}$ is convex open in $E$, and $S_{k, r}:=\omega_{r+1}\left(U_{0}^{k}\right) \prec R_{k, r}$. Let $P_{k, r}:=\omega_{r+1}\left(U^{k}\right)$.

Define complexes

$$
\begin{gathered}
\mathcal{R}_{r}:=\left\{R_{k, r}: r+1 \neq k \in H_{r}\right\}, \quad \mathcal{S}_{r}:=\left\{S_{k, r}: r+1 \neq k \in H_{r}\right\}, \\
\mathcal{P}_{r}:=\left\{P_{k, r}: r+1 \neq k \in H_{r}\right\} .
\end{gathered}
$$

By $(3), \mathcal{S}_{r} \prec \mathcal{R}_{r}$, and $\mathcal{R}_{r}$ consists of convex open sets.

Take any simplex $\Delta$ of $\mathcal{P}_{r}$. Since $\omega_{r+1}$ is injective, $\Delta$ is also a simplex of $\left\{U^{k}: r+1 \neq k \in H_{r}\right\}$; consequently, $\Delta$ is a simplex of $\mathcal{A}(r+1)$, and, as $r+1 \notin \Delta$, it must be a simplex of class I or class II. Hence $\sigma_{r+1}(\Delta)$ has already been defined over $A(r+1)^{\Delta}$ at (e) or (f). By (g), the $\mathcal{L C}$-sections $\sigma_{r+1}(\Delta)$ for simplices $\Delta$ of $\mathcal{P}_{r}$ agree on the sets $U_{0}^{k}$ for $r+1 \neq k \in H_{r}$ (which is $\mathcal{H}_{2}(r+1)$ for simplices of classes I and II), and coincide with $\tau$ on $U_{0}^{k}$ for $r+1 \neq k \in H_{r} \cap \Theta\left(\right.$ which is $\left.\mathcal{H}_{3}(r+1)\right)$.

The $\mathcal{L C}$-sections $\sigma_{r+1}(\Delta)$ may be translated into $\mathcal{L C}$-mappings $\mu_{r+1}(\Delta)$ : $P_{r}^{\Delta} \rightarrow Y\left(P_{r}^{\Delta}\right.$ is the star of $\Delta$ in the complex $\left.\mathcal{P}_{r}\right)$, by the formula

$$
\mu_{r+1}(\Delta):=p_{2} \circ \phi_{r+1} \circ \sigma_{r+1}(\Delta) \circ \omega_{r+1}^{-1} .
$$

The mappings $\mu_{r+1}(\Delta)$ fit together over the sets $S_{k, r}$, and also agree with $p_{2} \circ \phi_{r+1} \circ \tau \circ \omega_{r+1}^{-1}$ on $S_{k, r}$ when $r+1 \neq k \in H_{r} \cap \Theta$. Any simplex of $\mathcal{R}_{r}$ is $a$ fortiori a simplex of $\mathcal{P}_{r}$, and $\mathcal{R}_{r}$ consists of open convex sets (although $\mathcal{P}_{r}$ need not), so $[2,5.1]$ applies with $\mathcal{R}_{r}$ in place of " $\mathcal{A}$ ", $\mathcal{S}_{r}$ instead of " $\mathcal{B}$ ", the whole of $E$ as " $X$ ", and $\mu_{r+1}(\Delta)$ as " $f_{\Delta}$ "; hence, there is an $\mathcal{L} \mathcal{C}$-mapping $h_{r}: E \rightarrow Y$ which agrees with $\mu_{r+1}(\Delta)$ on $S_{k, r}$, for any simplex $\Delta$ of $\mathcal{R}_{r}$ and any vertex $k$ of $\Delta$. One may therefore use $h_{r}$ to define $\sigma_{r+1}(\Gamma)$ simultaneously for all simplices $\Gamma$ of $\mathcal{A}(r+1)$ of class III; for each such simplex $\Gamma, A(r+1)^{\Gamma} \subseteq O_{r+1}$ by $3.3(\mathrm{iv})$, and one may set, for any $x \in A(r+1)^{\Gamma}$,

$$
\sigma_{r+1}(\Gamma)(x):=\phi_{r+1}^{-1}\left(x, h_{r} \circ \omega_{r+1}(x)\right) .
$$

This gives an $\mathcal{L C}$-section of $\pi$ over $A(r+1)^{\Gamma}$, and is the restriction of the $\mathcal{L C}$-section over $O_{r+1}$ defined by the formula on the right-hand side.

(j) Let $\Gamma, \Delta$ be simplices of $\mathcal{A}(r+1)$, and $j \in \Gamma \cap \Delta$. If both $\Gamma$ and $\Delta$ are of class III, then (4) shows that $\sigma_{r+1}(\Gamma)$ and $\sigma_{r+1}(\Delta)$ agree on $U^{j}$ and not just on $U_{0}^{j}$. 
If only $\Gamma$ is of class III, then $\Gamma \ni r+1 \notin T_{r} \cup \Delta$. Given any $i \in \Delta$, $U^{i} \cap U^{j} \neq \emptyset$, and, as $j \in \Gamma, U^{j} \cap U^{r+1} \neq \emptyset$, so $i \in H_{r}$ and $i \neq r+1$. Hence, by (2), $A(r+1)^{\Delta} \subseteq O_{r+1}$, and $\Delta$ is also a simplex of $\mathcal{P}_{r}$ of class I or class II. However, $h_{r}$ was constructed to agree with $\mu_{r+1}(\Delta)$ over each $S_{k, r}$ for which $k \in \Delta$, and in particular over $S_{j, r}$; from the formulæ (4) and (5), this means that, for any $x \in U_{0}^{j}, \omega_{r+1}(x) \in S_{j, r}$,

$$
\begin{aligned}
\sigma_{r+1}(\Gamma)(x) & :=\phi_{r+1}^{-1}\left(x, h_{r} \circ \omega_{r+1}(x)\right) \\
& =\phi_{r+1}^{-1}\left(x, p_{2} \circ \phi_{r+1} \circ \sigma_{r+1}(\Delta)(x)\right)=\sigma_{r+1}(\Delta)(x),
\end{aligned}
$$

which, with (g), completes the proof of $\mathcal{H}_{2}(r+1)$. In the same way, $h_{r}$ was constructed to agree with $p_{2} \circ \phi_{r+1} \circ \tau \circ \omega_{r+1}^{-1}$ when $r+1 \neq k \in H_{r} \cap \Theta$, so that

$$
\sigma_{r+1}(\Gamma)\left|U_{0}^{k}=\tau\right| U_{0}^{k} .
$$

This establishes the case of $\mathcal{H}_{3}(r+1)$ not already settled by $(\mathrm{g})$. Consequently, the inductive hypotheses at stage $r+1$ have been ensured by the construction at stage $r$, and it may be iterated to produce a whole sequence of $\mathcal{L C}$-mappings $\sigma_{r}(\Delta)$.

(k) Recall the definition of $\sigma_{r}$ at $\mathcal{H}_{2}(r)$. The inductive construction above entails immediately that $\sigma_{r+1}\left|U_{0}^{i}=\sigma_{r}\right| U_{0}^{i}$ for any $i \in T_{r}$. (Take $\Delta:=\{i\}$ in (e).) Consequently, a section $\sigma$ of $\pi$ may be well defined by setting $\sigma$ to agree with $\sigma_{r}$ over $U_{0}^{i}$ when $i \in T_{r}$. Since $\bigcup_{r=1}^{\infty} T_{r}=J$ by (1), this defines $\sigma$ over $M$ (by 3.1(i)); $\sigma$ is an $\mathcal{L C}$-mapping, since each $\sigma_{r}$ is $\mathcal{L C}$ by construction; and $\mathcal{H}_{3}(r)$ evidently ensures that $\sigma\left|U_{0}(\Theta)=\tau\right| U_{0}(\Theta)$.

REMARK 3.7. The induction above is reminiscent of $[2,4.2]$, because of the necessity of maintaining consistency of each step of the construction with some fixed data (in this case $\tau$ is given, in $[2,4.2] f$ ). A curiosity of the proof is that the local extension $h_{r}$ does not need to be defined on the whole of $E$, but only on $\omega_{r+1}\left(O_{r+1}\right)$. However, this does not permit any useful weakening of the hypotheses.

As here stated, 3.6 fails to mention a set $X$ such as appears in [2, 5.1]. To incorporate it directly into a similar proposition, one would have to make clumsy hypotheses on the coordinate transformations of $M$. A relatively attractive consequence of that stronger version of $[2,5.1]$ is given later, 6.1.

In the simple result 3.8 that follows, the topology on $F$ is not really necessary - it is included only for consistency with Definition 2.3. With a little revision of the definitions, one could obtain the proposition for functions into any vector space. The proof of 3.8 is exactly as in 3.6 , with all mention of local trivializations removed.

There are also versions of 3.6 corresponding to $[2,5.5]$, which I shall not discuss here because they require some further structure on the manifold, and an extended version corresponding to $[2,4.6]$, mentioned after 3.12 . 
Proposition 3.8. Let $\mathcal{C}$ be an admissible category, and let $M$ be an $\mathcal{L C}$-manifold with locally convex Lindelöf model $E$. Let $Y$ be a convex set in the topological vector space $F$. Suppose $\left(\left\{U^{i}\right\},\left\{U_{0}^{i}\right\}, \Theta\right)$ is an extension system in $M$. Then, for any $\mathcal{L C}$-mapping $g: U(\Theta) \rightarrow F$ with image in $Y$, there exists an $\mathcal{L C}$-mapping $f: M \rightarrow F$ with image in $Y$ such that $f\left|U_{0}(\Theta)=g\right| U_{0}(\Theta)$.

Definition 3.9. Let $M$ be an $\mathcal{L C}$-manifold. Say that a set $X$ in $M$ is an $\mathcal{L C}$-extension core in $M$ (for $\mathcal{L C}$-sections of locally trivial $\mathcal{L C}$-fibrations with fibres $\mathcal{L C}$-diffeomorphic to open convex sets in topological vector spaces) if it is closed and, for any open neighbourhood $U$ of $X$ in $M$, there is an open neighbourhood $V$ of $X$ such that, for any $\mathcal{L C}$-section $\tau$ over $U$ of a locally trivial $\mathcal{L C}$-fibration $\pi: L \rightarrow M$ with fibre $\mathcal{L C}$-diffeomorphic to an open convex subset $Y$ of a locally convex space $F$, there is an $\mathcal{L C}$-section $\sigma$ of $\pi$ over $M$ for which $\sigma|V=\tau| V$.

This definition may be varied by considering different kinds of $\tau$ and $\sigma$, such as real-valued $\mathcal{L C}$-functions or sections of locally trivial $\mathcal{L C}$-fibrations with more general fibres. In the rest of the paper, I shall consider a condition which is sufficient to ensure that a set is an $\mathcal{L C}$-extension core either in the sense above, or for $\mathcal{L C}$-functions with values in a topological vector space.

Definition 3.10. The subset $X$ of the $\mathcal{D}$-manifold $M$ is simply $\mathcal{D}$ extensible, where $\mathcal{D}$ is an applicable category, if $X$ is closed in $M$ and, for any open cover $\mathfrak{V}$ of $M$ and open neighbourhood $V$ of $X$, there is a strong $\mathfrak{V}$-extension system $\left(\left\{\left(U^{j}, U_{0}^{j}\right): j \in J\right\}, \Theta\right)$ (see 3.5) such that $X \subseteq U_{0}(\Theta)$ and $U(\Theta) \subseteq V$.

REMARK 3.11. This condition involves only $X$ and $M$, and not a specific extension problem; to prove that $X$ is an $\mathcal{L C}$-extension core in a particular sense, it is commonly not necessary to demand strong extension systems or to allow arbitrary open covers $\mathfrak{V}$. The proof of 3.6 only requires the members of $\mathfrak{V}$ to admit $\mathcal{L C}$-local trivializations, which in some circumstances will exist over certain sets for any locally trivial fibration, for instance because of contractibility. In short, simple $\mathcal{L C}$-extensibility is often an excessively strong condition for $\mathcal{L C}$-extension cores of a given kind, but it works rather generally.

If $M$ is, as usual, $\mathrm{T}_{4}$, explicit mention of $V$ in 3.10 may seem superfluous, as one could consider the finer cover $\mathfrak{V}^{\prime}$ consisting of all the sets $V \cap W$ and $W \backslash M$ as $W$ varies over $\mathfrak{V}$. However, it might then be necessary to diminish $\Theta$ in order to ensure that $U(\Theta) \subseteq V$, and the removal of some element $\theta$ from $\Theta$ may invalidate 3.1(ii), since there is no reason why $U^{\theta}$ should meet only finitely many other sets $U^{\phi}$ for which $\phi \in \Theta$. If one were to add to 3.5 a requirement that $\Theta:=\left\{j \in J: U^{j} \cap X \neq \emptyset\right\}$, this objection ceases to 
apply and mention of $V$ in 3.10 could be suppressed. The proposed extra requirement is fairly natural and is true in the specific constructions of $\S 5$, but is not otherwise necessary.

Proposition 3.12. Suppose $\mathcal{C}$ is an admissible category (see 2.2), and $M$ is an $\mathcal{L C}$-manifold with locally convex Lindelöf model. If $X$ is a simply $\mathcal{L C}$-extensible subset of $M$, then $X$ is an $\mathcal{L C}$-extension core in $M$.

Furthermore, for any $\mathcal{L C}$-map $g: U \rightarrow F$, where $F$ is a topological vector space and $U$ is a neighbourhood of $X$ in $M$, there exists an $\mathcal{L C}$-map $f: M \rightarrow F$ such that $f|V=g| V$ (where $V \subseteq U$ is the neighbourhood of $X$ which is specified in Definition 3.9) and $f(M) \subseteq \operatorname{co}(g(U))$.

The section $\sigma$ or the mapping $f$ is obtained from $\tau$ or $g$ by a procedure of taking convex combinations of local extensions (see 3.6 and $[2$, passim]) in a way which does not depend on $\tau$ or $g$, but only on $X, U$ and $M$; hence, if the local extensions depend affine-linearly on $\tau$ or $g$, so do the global extensions. In this paper, the existence of linear local extension operators in the sense of $[2,4.4]$ is assured for $\mathcal{L C}$-sections or $\mathcal{L C}$-functions by linear algebra, since there are surjective linear restriction operators by definition.

My purpose now is to establish criteria for sets to be simply $\mathcal{L C}$-extensible. I shall concentrate my attention on manifolds which are modelled on normed spaces and $\mathrm{C}^{1}$ in the sense of Fréchet calculus; for them the condition $3.5\left(\mathrm{iv}^{\prime}\right)(\mathrm{b})$ is rather easily satisfied, because a $\mathrm{C}^{1}$ mapping deforms small norm-balls whilst approximately preserving convexity. This property is most easily described by Finsler structures. (There are other less natural categories $\mathcal{C}$ for which similar but more complicated procedures are possible).

\section{4. $\varepsilon$-flatness in Finsler manifolds}

Definition 4.1. Let $M$ be a $\mathrm{C}^{0}$ manifold modelled on a topological space $Y$. A chart for $M$ is then just a homeomorphism of an open set in $M$ with an open set in $Y$. Define a chart to be strong in $M$ if it is a chart $\psi: U \rightarrow Y$ (where $U$ is open in $M$ and $\psi(U)$ open in $Y$ ) such that, whenever $C$ is a closed set in $M$ and $C \subseteq U$, then $\psi(C)$ is closed in $Y$, and, conversely, whenever $D$ is a closed set in $Y$ which is included in $\psi(U)$, then $\psi^{-1}(D)$ is closed in $M$.

This definition is useful because charts may "misbehave near the boundary". In finite dimensions, the problem is customarily avoided by making use of compact rather than closed sets.

A $\mathrm{C}^{0}$ atlas $\mathfrak{A}$ for $M$ is hereditary if, whenever $\psi: U \rightarrow \psi(U) \subseteq Y$ is a chart belonging to $\mathfrak{A}$ and $V$ is an open subset of $U, \psi \mid V: V \rightarrow \psi(V)$ is also in $\mathfrak{A}$. 
Lemma 4.2. Suppose the spaces $X$ and $Y$ are both $\mathrm{T}_{4}$. Let $U$ be open in $X$ and $V$ in $Y$, and let $\psi: U \rightarrow V$ be a homeomorphism. If $C$ is a closed subset of $X$ included in $U$ and such that $\psi(C)$ is closed in $Y$, then there exists an open neighbourhood $W$ of $C$ in $U$ such that $\psi$ establishes a one-one correspondence between the closed sets of $X$ included in $W$ and the closed sets of $Y$ included in $\psi(W)$. Similarly, if $M$ is a regular $\mathrm{C}^{0}$ manifold, $M$ an open submanifold, and $\mathfrak{A}$ a hereditary atlas for $M$, the charts of $\mathfrak{A}$ which are strong in $M$ also form an atlas for $M$. Finally, the restriction of a chart strong in $M$ to an open subset of its domain is also a chart strong in $M$.

Proof. As $Y$ and $X$ are $\mathrm{T}_{4}$, take neighbourhoods $W_{1}$ of $C$ in $X$, and $W_{2}$ of $\psi(C)$ in $Y$, such that the closure $\bar{W}_{1}$ of $W_{1}$ in $X$ is within $U$ and the closure $\bar{W}_{2}$ of $W_{2}$ in $Y$ is within $\psi(U)$. Take $W:=W_{1} \cap \psi^{-1}\left(W_{2}\right)$. It is easy to check that $W$ has the required property. For the second assertion, it suffices to replace " $C$ " by an arbitrary point " $x$ ", and the argument then only requires regularity.

Definition 4.3. The category $\mathcal{D}$ is adequate if it is applicable (see 2.4), its objects are open subsets in normed spaces, and all its morphisms are $\mathrm{C}^{1}$ in the Fréchet sense.

For the rest of this section $M$ is to be a paracompact, but not necessarily separable, $\mathcal{D}$-manifold, where $\mathcal{D}$ is an adequate category and $M$ is modelled on the normed space $E$. ( $M$ and $E$ are therefore regular.) There is no need here for $\mathcal{D}$ to have a local extensibility property. $M$ admits a tangential Finsler structure $p$ (for the infinite-dimensional case, see, for instance, [10] or [1]), which induces a corresponding Finsler metric $d$. The metric ball of radius $r$ about $x \in M$ will be denoted by $B(x ; r)$. (In 3.6 a similar notation $B(r)$ was used with a quite different meaning). I shall write $p_{x}(\xi)$ or $p(x, \xi)$ to denote the Finsler norm of the vector $\xi$ in the tangent space $T_{x} M$ to $M$ at $x$.

Definition 4.4. Suppose that $\varepsilon \geq 0$. A $\mathcal{D}$-chart $\psi: U \rightarrow E$ of $M$ is $\varepsilon$-flat if it is strong in $M$ and, for any two points $y, z \in U$, and any $\xi \in E$,

$$
p_{y}\left(\left(T_{y} \psi\right)^{-1} \xi\right) \leq(1+\varepsilon) p_{z}\left(\left(T_{z} \psi\right)^{-1} \xi\right)
$$

LEMMA 4.5. Any subchart of an $\varepsilon$-flat $\mathcal{D}$-chart of $M$ is itself $\varepsilon$-flat. If $\varepsilon>0, \psi: U \rightarrow E$ is a $\mathcal{D}$-chart of $M$, and $x \in U$, then $x$ has a base of open neighbourhoods in $U$ on each of which the restriction of $\psi$ is $\varepsilon$-flat.

Proof. Any restriction to an open subset of a chart in $\mathfrak{A}$ satisfying (6) and strong in $M$ is also a chart strong in $M$, by 4.2 , and evidently still satisfies (6). It therefore suffices to prove the existence of one $\varepsilon$-flat neighbourhood of $x$. Take any strong chart $\psi: U \rightarrow E$, where $x \in U$, and then, using the definition of a tangential Finsler structure $([10]$ or $[1])$, let $V$ be 
an open neighbourhood of $x$ in $U$ such that, for all $y \in V$ and all $\xi \in E$,

$$
(1+\varepsilon)^{-1 / 2} p_{y}\left(\left(T_{y} \psi\right)^{-1} \xi\right) \leq p_{x}\left(\left(T_{x} \psi\right)^{-1} \xi\right) \leq(1+\varepsilon)^{1 / 2} p_{y}\left(\left(T_{y} \psi\right)^{-1} \xi\right) .
$$

Then $\psi \mid V$ is an $\varepsilon$-flat chart.

Lemma 4.6. Let $\omega: B(x ; R) \rightarrow E$ be a D-chart of $M$. Norm $E$ by

$$
\|\xi\|_{\omega, x}:=p_{x}\left(\left(T_{x} \omega\right)^{-1} \xi\right)
$$

and let $B_{\omega, x}^{\prime}(\eta ; \kappa)$ denote the ball of radius $\kappa$ about $\eta \in E$ with respect to $\|\xi\|_{\omega, x}$. If $\omega$ is $\varepsilon$-flat, then, for any $r \in[0, R)$,

$$
B_{\omega, x}^{\prime}\left(\omega(x) ;(1+\varepsilon)^{-1} r\right) \subseteq \omega(B(x ; r)) \subseteq B_{\omega, x}^{\prime}(\omega(x) ;(1+\varepsilon) r) .
$$

Proof. (6) ensures that, for each $y \in B(x ; r)$, and each $\eta \in T_{y} M$,

$$
(1+\varepsilon)^{-1}\left\|T_{y} \omega \cdot \eta\right\|_{\omega, x} \leq p_{y}(\eta) \leq(1+\varepsilon)\left\|T_{y} \omega \cdot \eta\right\|_{\omega, x} .
$$

(Take $\xi:=T_{y} \omega \cdot \eta$ to see this.) If $y \in B(x ; R)$ and $\delta>0$, and $\gamma(t)$, for $0 \leq t \leq 1$, is a piecewise $\mathrm{C}^{1}$ path in $B(x ; R)$ from $x$ to $y$ of $p$-length less than $d(x, y)+\delta$, then, from the first inequality of $(9), \omega \circ \gamma$ has \|\|$_{\omega, x}$-length not exceeding $(1+\varepsilon)(d(x, y)+\delta)$, and

$$
\|\omega(x)-\omega(y)\|_{\omega, x} \leq(1+\varepsilon)(d(x, y)+\delta) .
$$

Since $\delta$ may be arbitrarily small, the second inclusion of (8) follows.

Let $a \in E,\|a\|_{\omega, x}=1$. Define the ray $\gamma_{a}$ by $\gamma_{a}(t):=\omega(x)+t a$, for $t \geq 0$. Let

$$
\tau_{a}:=\sup \left\{t \geq 0: \gamma_{a}([0, t]) \subseteq \omega(B(x ; r))\right\} .
$$

Since $\omega(B(x ; r))$ is open in $E$, certainly $\tau_{a}>0$. Consider the lifted path

$$
\widehat{\gamma}_{a}:=\omega^{-1} \circ \gamma_{a}:\left[0, \tau_{a}\right) \rightarrow B(x ; r) .
$$

This is a $\mathrm{C}^{1}$ path without end-point. Its length in $M$ is

$$
\int_{0}^{\tau_{a}} p\left(\widehat{\gamma}_{a}(t),\left(T_{\widehat{\gamma}_{a}(t)} \omega\right)^{-1} a\right) d t \leq \int_{0}^{\tau_{a}}(1+\varepsilon)\|a\|_{\omega, x} d t=(1+\varepsilon) \tau_{a},
$$

by the second inequality of $(9)$. Thus $\widehat{\gamma}_{a}\left(\left[0, \tau_{a}\right)\right) \subseteq B\left(x ;(1+\varepsilon) \tau_{a}\right)$.

Suppose, if possible, that $(1+\varepsilon) \tau_{a}<r$. The closure $C$ of $B\left(x ;(1+\varepsilon) \tau_{a}\right)$ in $M$ is then included in $B(x ; r)$. As $\omega$ is a chart strong in $M$ (by Definition 4.4; see also 4.1), $\omega(C)$ is closed in $E$. Since $\widehat{\gamma}_{a}\left(\left[0, \tau_{a}\right)\right) \subseteq C$, certainly $\gamma_{a}\left(\left[0, \tau_{a}\right)\right) \subseteq \omega(C)$. Hence $\gamma_{a}\left(\tau_{a}\right) \in \omega(C) \subseteq \omega(B(x ; r))$, which is open in $E$, so $\gamma_{a}([0, t]) \subseteq \omega(B(x ; r))$ for some values of $t$ greater than $\tau_{a}$, contradicting (10). Therefore $(1+\varepsilon) \tau_{a} \geq r$. This means, by (10), that $t a \in \omega(B(x ; r))$ for $0 \leq t<(1+\varepsilon)^{-1} r$, and proves the result. 
Lemma 4.7. Let $\varepsilon \in[0, \sqrt{2}-1]$, and suppose that $\omega: B(x ; 2 \delta) \rightarrow E$ is an $\varepsilon$-flat $\mathcal{D}$-chart of $M$. Then, for any $y, z \in B(x ; \delta)$,

$$
(1+\varepsilon)^{-1}\|\omega(y)-\omega(z)\|_{\omega, x} \leq d(y, z) \leq(1+\varepsilon)\|\omega(y)-\omega(z)\|_{\omega, x} .
$$

Proof. Since $d(y, z)<2 \delta$ by the triangle inequality, $d(y, z)$ is the infimum of lengths of piecewise $\mathrm{C}^{1}$ paths shorter than $2 \delta$ between $y$ and $z$. Any such path must stay wholly within $B(x ; 2 \delta)$; otherwise it would join $y$ to a point $w$ outside $B(x ; 2 \delta)$, by a segment necessarily of length greater than $\delta$, and then $w$ to $z$, by a second segment of length more than $\delta$; thus, the whole path would have to be of length greater than $2 \delta$.

Let $\gamma:[0,1] \rightarrow M$ be a piecewise $\mathrm{C}^{1}$ path in $B(x ; 2 \delta) \subseteq M$. Introduce a norm in $E$ by (7). The Finsler length of $\gamma$ with respect to $p, \mathbb{L}(\gamma)$, is given by the formula

$$
\mathbb{L}(\gamma):=\int_{0}^{1} p_{\gamma(t)}\left(\gamma^{\prime}(t)\right) d t,
$$

and, by (9), it follows that

$$
\mathbb{L}(\gamma) \geq(1+\varepsilon)^{-1} \int_{0}^{1}\left\|T_{\gamma(t)} \omega \cdot \gamma^{\prime}(t)\right\|_{\omega, x} d t .
$$

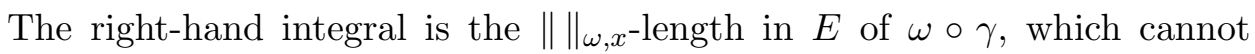
be less than $\|\omega(\gamma(1))-\omega(\gamma(0))\|_{\omega, x}$. (Rectilinear segments in $E$ minimize the norm-length of $\mathrm{C}^{1}$ paths between their end-points - from the triangle inequality and Riemann sums, for instance). Thus, for any piecewise $\mathrm{C}^{1}$ path $\gamma$ in $B(x ; 2 \delta)$,

$$
\mathbb{L}(\gamma) \geq(1+\varepsilon)^{-1}\|\omega(\gamma(1))-\omega(\gamma(0))\|_{\omega, x} .
$$

Since $d(y, z)$ is the infimum over such paths $\gamma$ between $z$ and $y$, the first of the stated inequalities is established.

Now join $y$ to $x$ by a piecewise $\mathrm{C}^{1}$ path $\beta:[0,1] \rightarrow M$ of Finsler-length less than $\delta$. Then

$$
\begin{aligned}
\|\omega(y)-\omega(x)\|_{\omega, x} & \leq \int_{0}^{1}\left\|(\omega \circ \beta)^{\prime}(t)\right\|_{\omega, x} d t=\int_{0}^{1}\left\|T_{\beta(t)} \omega \cdot \beta^{\prime}(t)\right\|_{\omega, x} d t \\
& \leq(1+\varepsilon) \int_{0}^{1} p_{\beta(t)}\left(\beta^{\prime}(t)\right) d t=(1+\varepsilon) \mathbb{L}(\beta)<(1+\varepsilon) \delta .
\end{aligned}
$$

The same holds for $\|\omega(z)-\omega(x)\|_{\omega, x}$. Hence, the straight-line segment $\theta$ between $\omega(y)$ and $\omega(z)$, defined by $\theta(t):=t \omega(z)+(1-t) \omega(y)$ for $0 \leq t \leq 1$, lies entirely within the open ball of radius $(1+\varepsilon) \delta$ about $\omega(x)$ in $E$. By hypothesis, $(1+\varepsilon) \delta \leq 2 \delta /(1+\varepsilon)$; hence, from 4.6, all points of the segment 
lie in $\omega(B(x ; 2 \delta))$, so that the path $\zeta:=\omega^{-1} \circ \theta$ joining $y$ to $z$ is defined and $\mathrm{C}^{1}$ in $B(x ; 2 \delta)$. Apply $(9)$ :

$$
\begin{aligned}
d(y, z) & \leq \mathbb{L}(\zeta)=\int_{0}^{1} p_{\zeta(t)}\left(\zeta^{\prime}(t)\right) d t \leq(1+\varepsilon) \int_{0}^{1}\left\|T_{\zeta(t)} \omega \cdot \zeta^{\prime}(t)\right\|_{\omega, x} d t \\
& =(1+\varepsilon) \int_{0}^{1}\left\|\theta^{\prime}(t)\right\|_{\omega, x} d t=(1+\varepsilon)\|\omega(z)-\omega(y)\|_{\omega, x},
\end{aligned}
$$

and this is exactly the second inequality required.

Definition 4.8. Given $t \in \mathbb{R}$ and $a \in E$, the affine expansion by $t$ about $a$ is the mapping $A(t, a): E \rightarrow E: \xi \mapsto t \xi+(1-t) a$.

REMARK 4.9. If $C$ is a convex subset of $E$ and $a \in C$, then, whenever $t>1, C \prec A(t, a) C$.

Corollary 4.10. Let $r, R, \delta, \varepsilon$ be positive numbers such that $\varepsilon \leq \sqrt{2}-1$ and

$$
2(1+\varepsilon)^{3}(r+\delta) \leq R
$$

Then, if $\omega: B(x ; R) \rightarrow E$ is an $\varepsilon$-flat chart and $y \in B(x ; r)$,

$$
A(1+\varepsilon, \omega(y)) \operatorname{co}\left(\omega ( B ( y ; \delta ) ) \subseteq \omega \left(B\left(y ;(1+\varepsilon)^{3} \delta\right) .\right.\right.
$$

Proof. Certainly $B(y ; \delta) \subseteq B(x ; r+\delta)$, and $2(r+\delta) \leq R$. By the first inequality of $4.7, \omega(B(y ; \delta)) \subseteq B_{\omega, x}^{\prime}(\omega(y) ;(1+\varepsilon) \delta)$, and, therefore,

$$
\operatorname{co}\left(\omega(B(y ; \delta)) \subseteq B_{\omega, x}^{\prime}(\omega(y) ;(1+\varepsilon) \delta) .\right.
$$

But, by $4.6, \omega(y) \in B_{\omega, x}^{\prime}(\omega(x) ;(1+\varepsilon) r)$, and so

$$
\begin{aligned}
B_{\omega, x}^{\prime}\left(\omega(y) ;(1+\varepsilon)^{2} \delta\right) & \subseteq B_{\omega, x}^{\prime}\left(\omega(x) ;(1+\varepsilon)^{2}(r+\delta)\right) \\
& \subseteq \omega\left(B\left(x ;(1+\varepsilon)^{3}(r+\delta)\right)\right) .
\end{aligned}
$$

So, if $\zeta \in B_{\omega, x}^{\prime}\left(\omega(y) ;(1+\varepsilon)^{2} \delta\right)$, there is some $z \in B\left(x ;(1+\varepsilon)^{3}(r+\delta)\right)$ such that $\omega(z)=\zeta$, and, by the second inequality of $4.7, z \in B\left(y ;(1+\varepsilon)^{3} \delta\right)$. Hence

$$
B_{\omega, x}^{\prime}\left(\omega(y) ;(1+\varepsilon)^{2} \delta\right) \subseteq \omega\left(B\left(y ;(1+\varepsilon)^{3} \delta\right)\right) .
$$

From (11),

$$
\begin{aligned}
A(1+\varepsilon, \omega(y)) \operatorname{co}(B(\omega(y) ; \delta)) & \subseteq A(1+\varepsilon, \omega(y)) B_{\omega, x}^{\prime}(\omega(y) ;(1+\varepsilon) \delta) \\
& =B_{\omega, x}^{\prime}\left(\omega(y) ;(1+\varepsilon)^{2} \delta\right),
\end{aligned}
$$

which, with (12), proves the result.

Definition 4.11. Let $\mathfrak{A}$ be a hereditary $\mathcal{D}$-atlas for $M$. The modulus of $\varepsilon$-flatness of $M$ with respect to $\mathfrak{A}$ and $p$ is the function $\varrho_{\varepsilon}: M \rightarrow \mathbb{R}$ defined as follows. For each $x \in N$, consider the class of positive numbers $\delta$ such 
that $B(x ; \delta)$ is the domain of an $\varepsilon$-flat chart of $\mathfrak{A}$. This class has a supremum $\varrho_{\varepsilon}^{\prime}(x)$, which may be $\infty$ or $-\infty$. Set $\varrho_{\varepsilon}(x):=\min \left(1, \varrho_{\varepsilon}^{\prime}(x)\right)$.

LEMMA 4.12. If $\varepsilon>0$, the modulus of $\varepsilon$-flatness $\varrho_{\varepsilon}$ with respect to the Finsler structure $p$ and the atlas $\mathfrak{A}$ takes strictly positive values, and satisfies the Lipschitz condition that, for any $x, y \in N,\left|\varrho_{\varepsilon}(x)-\varrho_{\varepsilon}(y)\right| \leq d(x, y)$.

Proof. If $\psi: U \rightarrow E$ is a chart of $\mathfrak{A}$, where $x \in U$, then by 4.5 there is an open neighbourhood $V$ of $x$ in $U$ such that $\psi \mid V$ is $\varepsilon$-flat. Take $\delta>0$ such that $B(x ; \delta) \subseteq V$; then $\varrho_{\varepsilon}^{\prime}(x) \geq \delta$. This proves the first assertion.

Suppose $d(x, y)<\varrho_{\varepsilon}^{\prime}(x)$; let $\kappa \in\left(d(x, y), \varrho_{\varepsilon}^{\prime}(x)\right)$. By hypothesis, there is an $\varepsilon$-flat chart of $\mathfrak{A}, \phi: B(x ; \kappa) \rightarrow E$. Now $B(y ; \kappa-d(x, y)) \subseteq B(x ; \kappa)$; hence, $\phi \mid B(y ; \kappa-d(x, y))$ is also $\varepsilon$-flat and in $\mathfrak{A}$, and $\varrho_{\varepsilon}^{\prime}(y) \geq \kappa-d(x, y)$. Since $\kappa$ was arbitrary in $\left(d(x, y), \varrho_{\varepsilon}^{\prime}(x)\right), \varrho_{\varepsilon}^{\prime}(y) \geq \varrho_{\varepsilon}^{\prime}(x)-d(x, y)$. This is also trivially true if $d(x, y) \geq \varrho_{\varepsilon}^{\prime}(x)$.

Consequently, either $\varrho_{\varepsilon}^{\prime}(x)$ is infinite for all $x$, or it is finite for all $x$. In the second case, by symmetry, $\left|\varrho_{\varepsilon}^{\prime}(x)-\varrho_{\varepsilon}^{\prime}(y)\right| \leq d(x, y)$. The desired result for $\varrho_{\varepsilon}(x)$ follows.

LEMmA 4.13. Suppose $\sigma: M \rightarrow[0, \infty)$ is a function satisfying the Lipschitz condition that $|\sigma(x)-\sigma(y)| \leq d(x, y)$ for all $x, y \in M$. Let $0 \leq \alpha<1$. If $x_{i} \in M$ for $i=0,1,2,3,4$, and, for $i=0,1,2,3$,

$$
B\left(x_{i} ; \alpha \sigma\left(x_{i}\right)\right) \cap B\left(x_{i+1} ; \alpha \sigma\left(x_{i+1}\right)\right) \neq \emptyset,
$$

then, for $0 \leq i \leq 4$,

$$
\sigma\left(x_{i}\right)<\left(\frac{1+\alpha}{1-\alpha}\right)^{i} \sigma\left(x_{0}\right) \quad \text { and } \quad B\left(x_{4} ; \alpha \sigma\left(x_{4}\right)\right) \subseteq B\left(x_{0} ; \beta \sigma\left(x_{0}\right)\right),
$$

where

$$
\beta:=\alpha\left(\frac{9+4 \alpha+14 \alpha^{2}+4 \alpha^{3}+\alpha^{4}}{(1-\alpha)^{4}}\right)
$$

Proof. Certainly $d\left(x_{0}, x_{1}\right)<\alpha \sigma\left(x_{0}\right)+\alpha \sigma\left(x_{1}\right)$, so that from the Lipschitz condition,

$$
\sigma\left(x_{1}\right)<\sigma\left(x_{0}\right)+\alpha \sigma\left(x_{0}\right)+\alpha \sigma\left(x_{1}\right),
$$

and consequently $\sigma\left(x_{1}\right)<((1+\alpha) /(1-\alpha)) \sigma\left(x_{0}\right)$, and similarly $\sigma\left(x_{2}\right)<$ $((1+\alpha) /(1-\alpha)) \sigma\left(x_{1}\right)$ and so on. Hence, if $y \in B\left(x_{4} ; \alpha \sigma\left(x_{4}\right)\right)$,

$$
d\left(x_{0}, y\right)<\alpha\left(1+2 \frac{1+\alpha}{1-\alpha}+2\left(\frac{1+\alpha}{1-\alpha}\right)^{2}+2\left(\frac{1+\alpha}{1-\alpha}\right)^{3}+2\left(\frac{1+\alpha}{1-\alpha}\right)^{4}\right) \sigma(x)
$$

which is, in effect, the result stated.

This result trivially implies similar statements for smaller numbers of balls. 


\section{Simply $\mathcal{D}$-extensible sets}

Proposition 5.1. Let $M$ be a paracompact $\mathcal{D}$-manifold, where $\mathcal{D}$ is an applicable category (see 2.4). Let $X$ be a closed subset of $M$, and let $K$ be a closed locally compact subset of $M$. If $X$ is simply $\mathcal{D}$-extensible, then so is $X \cup K$.

A finite-dimensional submanifold, in the sense of 2.5, of a (topological) manifold $M$ modelled on a topological vector space (one takes $\mathcal{D}$ in 2.5 to consist of all continuous maps of open sets) is necessarily a closed locally compact set in $M$. (The corresponding subspace of the model is closed, being finite-dimensional.)

Proof. (a) Suppose that $V$ is an open neighbourhood of $X \cup K$ and $\mathfrak{V}$ is an open cover of $M$. As $K$ is closed in $M$, it is paracompact in itself. As it is locally compact, $K=\bigcup_{\beta \in B} K_{\beta}$, where $\left\{K_{\beta}: \beta \in B\right\}$ is a locally finite family of compact sets in $M$ whose relative interiors cover $K$; the index set $B$ may be assumed not to contain 0. By 5.1.19 of [6], there are open sets $W_{\beta} \supseteq K_{\beta}$, for each $\beta \in B$, and $W_{0} \supseteq X$, such that $\mathcal{T}:=\left\{W_{\beta}: \beta \in B \cup\{0\}\right\}$ is locally finite in $M$. I may also suppose that $W_{\beta} \subseteq V$ for each $\beta \in B_{0}:=B \cup\{0\}$. Write $K_{0}$ for $X$.

(b) If $x \notin X \cup K$, let $U(x)$ be an open neighbourhood of $x$ in $M$ which does not meet $X \cup K$ and meets only finitely many $W_{\beta}$. If $x \in X \cup K$, let

$$
B(x):=\left\{\beta \in B_{0}: x \in K_{\beta}\right\},
$$

which is finite, and set $U(x):=\left(\bigcap_{\beta \in B(x)} W_{\beta}\right) \backslash\left(\bigcup_{\gamma \in B \backslash B(x)} K_{\gamma}\right)$, which is also an open neighbourhood of $x$. In this way $U(x)$ has the property for each $x$ that it meets only finitely many $W_{\beta}$, and, if it meets $K_{\beta}$, then $x \in K_{\beta}$ and $U(x) \subseteq W_{\beta}$.

Let $\mathcal{S}:=\{U(x): x \in M\}$, an open cover of $M$. Let $\mathfrak{W}$ be an open cover of $M$ which simultaneously refines $\mathfrak{V}$ and $\mathcal{S}$. By hypothesis, there is a $\mathfrak{W}$-extension system $\left(\left\{\left(U^{j}, U_{0}^{j}\right): j \in J\right\}, \Theta\right)$ in $M$ such that $X \subseteq U_{0}(\Theta)$ and $U(\Theta) \subseteq W$.

(c) For each $\beta \in B$, there is, as $K_{\beta}$ is compact, a finite subset $\Phi(\beta)$ of $J$ such that $K_{\beta} \subseteq \bigcup_{j \in \Phi(\beta)} U_{0}^{j}$. By omitting indices for which it is false, I may suppose that

$$
U_{0}^{k} \cap K_{\beta} \neq \emptyset \quad \text { for each } k \in \Phi(\beta) .
$$

Let $\Phi:=\bigcup_{\beta \in B} \Phi(\beta)$, and set $\Theta^{\prime}:=\Theta \cup \Phi$.

(d) Take any $\psi \in J \backslash \Theta$. Then $\left\{\theta \in \Theta: U^{\psi} \cap U^{\theta} \neq \emptyset\right\}$ is finite by 3.1(ii). On the other hand, if $\phi \in \Phi, U^{\phi} \subseteq U(x)$ for some $x \in M$ (as $\left\{U^{j}\right\}$ refines $\mathcal{S}$ ); but, by (13), $U^{\phi} \cap K \neq \emptyset$, and therefore $U(x) \cap K \neq \emptyset$. From (b), $U(x) \subseteq W_{\beta}$ for each $\beta \in B$ such that $U(x) \cap K_{\beta} \neq \emptyset$. Hence, if $\phi \in \Phi(\beta)$, 
$U^{\phi} \subseteq U(x) \subseteq W_{\beta}$, and, if $U^{\psi} \cap U^{\phi} \neq \emptyset$, necessarily $U^{\psi} \cap W_{\beta} \neq \emptyset$. But, again because $\left\{U^{j}\right\}$ refines $\mathcal{S}$, there are only finitely many such $\beta$ (see (b)). Ergo, $\phi$ must be in one of finitely many finite sets $\Phi(\beta)$, and $\left\{\phi \in \Phi: U^{\phi} \cap U^{\psi} \neq \emptyset\right\}$ is finite.

(e) The pair $\left(\left\{\left(U^{j}, U_{0}^{j}\right): j \in J\right\}, \Theta^{\prime}\right)$ clearly satisfies 3.1(i). I have just shown that, if $\psi \in J \backslash \Theta^{\prime}$ (indeed, if $\left.\psi \in J \backslash \Theta\right), U^{\psi} \cap U^{j} \neq \emptyset$ for only finitely many $j \in \Theta^{\prime}$, which is 3.1(ii). As for 3.1(iii) and 3.5(iv'), they are evidently still true.

This result would remain true if 3.3 were substituted for 3.5 in Definition 3.10 (just read 3.3(iv) for $3.5\left(\mathrm{iv}^{\prime}\right)$ ). Note that $\mathcal{D}$ need only be applicable (cf. 5.3 below). However, even the null set has not yet been proved to be simply $\mathcal{D}$-extensible.

One might on instinct expect a converse to Proposition 5.1, stating that, if $X \cup K$ is simply $\mathcal{D}$-extensible, $X$ is closed, and $K$ is closed locally compact in $M$, then $X$ is simply $\mathcal{D}$-extensible. This is not obvious, and probably false for the reasons noted after 3.10.

Lemma 5.2. Let $\mathcal{D}$ be an adequate category (see 4.3), and let $M$ be a $\mathcal{D}$-manifold, furnished with a Finsler structure $p$ and induced metric $d$ and modelled on the normed space $E$, with a $\mathcal{D}$-submanifold $N$ modelled on the closed linear subspace $F$ of $E$ (see 2.5). Metric balls are with reference to the metric d. Suppose $x \in N$, and $\omega: B\left(x ; \frac{24}{25} \varrho_{1 / 5}(x)\right) \rightarrow E$ is a $\frac{1}{5}$-flat $\mathcal{D}$-submanifold chart for $N$. Then $\omega$ and $p$ induce a norm \|\|$_{\omega, x}$ on $E$, as at (7) of 4.6. Let $\pi: E \rightarrow E / F$ be the quotient map, and let \|\|$_{\omega, x}^{\prime \prime}$ denote the quotient norm on $E / F$. Then, if $y \in B\left(x ; \frac{1}{6} \varrho_{\varepsilon}(x)\right)$,

$$
\frac{5}{6}\|\pi \circ \omega(y)\|_{\omega, x}^{\prime \prime} \leq d(y, N) \leq \frac{6}{5}\|\pi \circ \omega(y)\|_{\omega, x}^{\prime \prime},
$$

where, as usual, $d(y, N):=\inf \{d(y, z): z \in N\}$ (and similarly for other subsets of $M)$.

Proof. For notational convenience, write $\varepsilon:=\frac{1}{5}$. Recall from 4.6 that $B_{\omega, x}^{\prime}(\eta ; \kappa)$ denotes a ball in $E$ with respect to $\|\xi\|_{\omega, x}$. If $y \in B\left(x ; \frac{1}{6} \varrho_{\varepsilon}(x)\right)$, then $d(y, N)<\frac{1}{6} \varrho_{\varepsilon}(x)$. Now, $d(y, z)>\frac{1}{6} \varrho_{\varepsilon}(x)$ for any $z \notin B\left(x ; \frac{1}{3} \varrho_{\varepsilon}(x)\right)$, and therefore $d(y, N)$ may be calculated as the infimum of the distances $d(y, z)$ for points $z \in N \cap B\left(x ; \frac{1}{3} \varrho_{\varepsilon}(x)\right)$. For any such $z, 4.7$ implies that $\|\omega(y)-\omega(z)\|_{\omega, x} \leq \frac{6}{5} d(y, z)$. Taking infima over all choices of $z$, I find that $\|\pi \circ \omega(y)\|_{\omega, x}^{\prime \prime} \leq \frac{6}{5} d(y, N)$, the first inequality of (14).

By $4.6, \omega\left(B\left(x ; \frac{1}{6} \varrho_{\varepsilon}(x)\right)\right) \subseteq B_{\omega, x}^{\prime}\left(\omega(x) ; \frac{1}{5} \varrho_{\varepsilon}(x)\right)$. As a consequence,

$$
\|\pi \circ \omega(y)\|_{\omega, x}^{\prime \prime}<\frac{1}{5} \varrho_{\varepsilon}(x) .
$$

If $\zeta \in F$ and, for some positive $\delta$,

$$
\|\omega(y)-\zeta\|_{\omega, x}<\|\pi \circ \omega(y)\|_{\omega, x}^{\prime \prime}+\delta<\frac{1}{5} \varrho_{\varepsilon}(x),
$$


then $\|\zeta\|_{\omega, x}<\frac{2}{5} \varrho_{\varepsilon}(x)$, and, once again applying to $4.6, \zeta=\omega(w)$ for some $w \in N \cap B\left(x ; \frac{12}{25} \varrho_{\varepsilon}(x)\right)$. By 4.7 ,

$$
d(y, N) \leq d(y, w) \leq \frac{6}{5}\|\omega(y)-\zeta\|_{\omega, x} \leq \frac{6}{5}\left(\|\pi \circ \omega(y)\|_{\omega, x}^{\prime \prime}+\delta\right) .
$$

Since $\delta$ is arbitrarily small, $d(y, N) \leq \frac{6}{5}\|\pi \circ \omega(y)\|_{\omega, x}^{\prime \prime}$, which is the second inequality of (14).

Proposition 5.3. Let $M$ be a separable metrizable $\mathcal{D}$-manifold modelled on the normed space $E$, where $\mathcal{D}$ is an adequate category. Let $\left\{N_{\beta}: \beta \in B\right\}$ be a discrete family of $\mathcal{D}$-submanifolds of $M$, where, for each $\beta \in B, N_{\beta}$ is modelled on the closed linear subspace $F_{\beta}$ of $E$. Then $N:=\bigcup_{\beta \in B} N$ is a simply $\mathcal{D}$-extensible subset of $M$.

Proof. Since $M$ is paracompact, it admits a Finsler structure $p$ which, in turn, induces a metric $d$ (with respect to which metric balls are defined). Suppose given an open cover $\mathfrak{V}$ of $M$ and an open neighbourhood $V$ of $N$ in $M$. Let $\mathfrak{A}$ be the hereditary $\mathcal{D}$-atlas for $M$ consisting of $\mathcal{D}$-charts $\phi: U \rightarrow E$ such that

(i) $U \cap N_{\beta} \neq \emptyset$ for at most one index $\beta$,

(ii) $\phi$ is a $\mathcal{D}$-submanifold chart for $N_{\beta}$, for all $\beta \in B$,

(iii) there is a $W \in \mathfrak{V}$ such that either $U \subseteq W \cap V$ or $U \subseteq W \backslash N$.

Since $\left\{N_{\beta}\right\}$ is a discrete family, these charts do form an atlas. Indeed, if $x \notin N$, take any $\mathcal{D}$-chart about $x$, and restrict it to an open neighbourhood $U$ of $x$ such that $U \cap N=\emptyset$ and $U \subseteq W$ for some $W \in \mathfrak{V}$; if $x \in N_{\beta}$, take any $\mathcal{D}$-submanifold chart for $N_{\beta}$ about $x$ and restrict its domain to an open neighbourhood $U$ of $x$ such that $U \cap N_{\gamma}=\emptyset$ for any $\gamma \neq \beta$ and $U \subseteq W \cap V$ for some $W \in \mathfrak{V}$.

As in 5.2 , take $\varepsilon:=\frac{1}{5}$, and let $\varrho_{\varepsilon}$ be the modulus of $\varepsilon$-flatness with respect to $p$ and $\mathfrak{A}$ (see 4.11 ).

(a) The open cover $\left\{B\left(x ; \frac{1}{120} \varrho_{\varepsilon}(x)\right): x \in N\right\}$ of $N$ has a countable subcover, $\left\{B\left(x_{n} ; \frac{1}{120} \varrho_{\varepsilon}\left(x_{n}\right)\right): n \in \mathbb{N}\right\}$. For each $n \in \mathbb{N}$, let

$$
\left\{\begin{array}{l}
U_{0}^{n}:=B\left(x_{n} ; \frac{1}{120} \varrho_{\varepsilon}\left(x_{n}\right)\right) \cap\left\{y \in M: d(y, N)<\frac{1}{240} n^{-1} \varrho_{\varepsilon}\left(x_{n}\right)\right\}, \\
U^{n}:=B\left(x_{n} ; \frac{1}{60} \varrho_{\varepsilon}\left(x_{n}\right)\right) \cap\left\{y \in M: d(y, N)<\frac{1}{120} n^{-1} \varrho_{\varepsilon}\left(x_{n}\right)\right\} .
\end{array}\right.
$$

Since $B\left(x_{n} ; \frac{1}{120} \varrho_{\varepsilon}\left(x_{n}\right)\right) \cap N=U_{0}^{n} \cap N,\left\{U_{0}^{n}\right\}$ remains an open cover of $N$. By the construction of $\mathfrak{A}$ and $\varrho_{\varepsilon}$, either $B\left(x_{n} ; \frac{1}{2} \varrho_{\varepsilon}\left(x_{n}\right)\right) \subseteq M \backslash N$ (which is false) or $B\left(x_{n} ; \frac{1}{2} \varrho_{\varepsilon}\left(x_{n}\right)\right) \subseteq V$ for each $n$, so that $\bigcup_{n \in \mathbb{N}} U^{n} \subseteq V$, as required by 3.10 .

$N$ being closed, $d(x, N)>0$ for each $x \in M \backslash N$. Cover $M \backslash N$ by the balls $B\left(x ; \frac{1}{120} \min \left(\varrho_{\varepsilon}(x), d(x, N)\right)\right)$, where $x$ varies over $M \backslash N$. There is a countable subcover, whose index set $\Psi$ may be chosen to be disjoint from $\mathbb{N}$, 
$\left\{B\left(x_{\psi} ; \frac{1}{120} \min \left(\varrho_{\varepsilon}\left(x_{\psi}\right), d\left(x_{\psi}, N\right)\right)\right): \psi \in \Psi\right\} ;$ define $\Theta:=\mathbb{N}, J:=\Theta \cup \Psi$, so that $x_{j} \in N$ when and only when $j \in \Theta$, and set, for each $\psi \in \Psi$,

$$
\left\{\begin{array}{l}
U_{0}^{\psi}:=B\left(x_{\psi} ; \frac{1}{120} \min \left(\varrho_{\varepsilon}\left(x_{\psi}\right), d\left(x_{\psi}, N\right)\right)\right), \\
U^{\psi}:=B\left(x_{\psi} ; \frac{1}{60} \min \left(\varrho_{\varepsilon}\left(x_{\psi}\right), d\left(x_{\psi}, N\right)\right)\right) .
\end{array}\right.
$$

The condition 3.1(i) is satisfied, and, for any $j \in J, U^{j} \subseteq B\left(x_{j} ; \frac{1}{60} \varrho_{\varepsilon}\left(x_{j}\right)\right)$.

(b) Given $\psi \in \Psi$, (16) shows that, for any $z \in U^{\psi}$,

$$
d(z, N) \geq d\left(x_{\psi}, N\right)-\frac{1}{60} d\left(x_{\psi}, N\right)=\frac{59}{60} d\left(x_{\psi}, N\right) .
$$

On the other hand, if $z \in U^{n}$, (15) implies that $d(z, N)<\frac{1}{120} n^{-1}$, since $\varrho_{\varepsilon}\left(x_{n}\right) \leq 1$ by Definition 4.11 . Hence, $U^{\psi} \cap U^{n} \neq \emptyset$ only if $\frac{1}{120} n^{-1}>\frac{59}{60} d\left(y_{\psi}, N\right)$, and so for only finitely many $n$. This proves 3.1(ii).

(c) Suppose that $\psi \in \Psi$. There are two possibilities. The first is that, for any $r, s \in J$ such that $U^{\psi} \cap U^{r} \neq \emptyset \neq U^{r} \cap U^{s}$, necessarily $s \in \Psi$. This means, in particular, that the sets appearing in $3.5\left(\mathrm{iv}^{\prime}\right)(\mathrm{b})$ are all metric balls of the form (16). By 4.13, $U^{s} \subseteq B\left(x_{\psi} ; \frac{1}{6} \varrho_{\varepsilon}\left(x_{\psi}\right)\right)$. By Definition 4.11, there is some $\varepsilon$-flat $\mathcal{D}$-chart with domain $B\left(x_{\psi} ; \frac{2}{3} \varrho_{\varepsilon}\left(x_{\psi}\right)\right)$, and I choose such a chart as $\omega_{\psi}$, taking $O_{\psi}:=B\left(x_{\psi} ; \frac{2}{3} \varrho_{\varepsilon}\left(x_{\psi}\right)\right)$. Then $3.5\left(\mathrm{iv}^{\prime}\right)(\mathrm{a})$ is satisfied automatically.

(d) Suppose in the case (c) that $k, l \in J$ and $U^{\psi} \cap U^{k} \neq \emptyset \neq U^{k} \cap U^{l}$. From 4.13, $\varrho_{\varepsilon}\left(x_{l}\right)<\left(\frac{61}{59}\right)^{2} \varrho_{\varepsilon}\left(x_{\psi}\right)$. In 4.10 take $y:=x_{l}, x:=x_{\psi}$, and suppose

$$
R:=\frac{2}{3} \varrho_{\varepsilon}\left(x_{\psi}\right), \quad r:=\frac{1}{6} \varrho_{\varepsilon}\left(x_{\psi}\right), \quad 0<\delta \leq \frac{1}{120} \varrho_{\varepsilon}\left(x_{l}\right) .
$$

The hypothesis of 4.10 is satisfied, because

So

$$
2\left(\frac{6}{5}\right)^{3}\left(\frac{1}{6}+\left(\frac{61}{59}\right)^{2} \frac{1}{120}\right) \varrho_{\varepsilon}\left(x_{\psi}\right) \leq \frac{2}{3} \varrho_{\varepsilon}\left(x_{\psi}\right) .
$$

$$
A\left(\frac{6}{5}, \omega_{\psi}\left(x_{l}\right)\right) \operatorname{co}\left(\omega_{\psi}\left(B\left(x_{l} ; \delta\right)\right)\right) \subseteq \omega_{\psi}\left(B\left(x_{l} ;\left(\frac{6}{5}\right)^{3} \delta\right)\right) \subseteq \omega_{\psi}\left(B\left(x_{l} ; 2 \delta\right)\right) .
$$

If, in particular, $\delta:=\frac{1}{120} \min \left(\varrho_{\varepsilon}\left(x_{l}\right), d\left(x_{l}, N\right)\right)$, then

$$
A\left(\frac{6}{5}, \omega_{\psi}\left(x_{l}\right)\right) \operatorname{co}\left(\omega_{\psi}\left(U_{0}^{l}\right)\right) \subseteq \omega_{\psi}\left(U^{l}\right) .
$$

(e) The second possibility is that there are some $r \in J$ and $\theta \in \Theta$ such that $U^{\psi} \cap U^{r} \neq \emptyset \neq U^{r} \cap U^{\theta}$. (Here, $U^{\theta}$ is not defined as a metric ball; see (15). Indeed, (14) shows it usually is not one.) In this case, I choose such a $\theta$ and $r$. Certainly $x_{\theta} \in N_{\beta}$ for some $\beta \in B$. There is an $\varepsilon$-flat $\mathcal{D}$-submanifold chart for $N_{\beta}$ with domain $B\left(x_{\theta} ; \frac{24}{25} \varrho_{\varepsilon}\left(x_{\theta}\right)\right)$; I take one such chart to be $\omega_{\psi}$, letting $O_{\psi}$ be $B\left(x_{\theta} ; \frac{24}{25} \varrho_{\varepsilon}\left(x_{\theta}\right)\right)$, which again satisfies $3.5\left(\mathrm{iv}^{\prime}\right)(\mathrm{a})$.

If $y \in B\left(x_{\theta} ; \frac{1}{6} \varrho_{\varepsilon}\left(x_{\theta}\right)\right)$, then $d\left(y, N_{\gamma}\right)>\frac{5}{6} \varrho_{\varepsilon}\left(x_{\theta}\right)$ for any $\gamma \neq \theta$, since, by the definition of $\varrho_{\varepsilon}, B\left(x_{\theta} ; d\left(y, x_{\theta}\right)+\frac{5}{6} \varrho_{\varepsilon}\left(x_{\theta}\right)\right) \cap N_{\gamma}=\emptyset$. Therefore, $d(y, N)=$ $d\left(y, N_{\beta}\right)$. 
(f) Suppose $k, l \in J$ and $U^{\psi} \cap U^{k} \neq \emptyset \neq U^{k} \cap U^{l}$. By 4.13 (this time with a full chain of five sets), $U^{l} \subseteq B\left(x_{\theta} ; \frac{1}{6} \varrho_{\varepsilon}\left(x_{\theta}\right)\right)$. If $l \in \Psi$, note that

$$
2\left(\frac{6}{5}\right)^{3}\left(\frac{1}{6}+\left(\frac{61}{59}\right)^{4} \frac{1}{120}\right) \varrho_{\varepsilon}\left(x_{\psi}\right) \leq \frac{24}{25} \varrho_{\varepsilon}\left(x_{\psi}\right),
$$

so that 4.10 applies again (compare (d)) and

$$
A\left(\frac{6}{5}, \omega_{\psi}\left(x_{l}\right)\right)\left(\operatorname{co}\left(\omega_{\psi}\left(U_{0}^{l}\right)\right)\right) \subseteq \omega_{\psi}\left(U^{l}\right) .
$$

(g) If $l \in \mathbb{N}=\Theta$, take $\delta:=\frac{1}{120} \varrho_{\varepsilon}\left(x_{l}\right)$ in 4.10, and one deduces that

$$
\begin{aligned}
A\left(\frac{6}{5}, \omega_{\psi}\left(x_{l}\right)\right) \operatorname{co}\left(\omega_{\psi}\left(U_{0}^{l}\right)\right) & \subseteq A\left(\frac{6}{5}, \omega_{\psi}\left(x_{l}\right)\right) \operatorname{co}\left(\omega_{\psi}\left(B\left(x_{l} ; \frac{1}{120} \varrho_{\varepsilon}\left(x_{l}\right)\right)\right)\right) \\
& \subseteq \omega_{\psi}\left(B\left(x_{l} ; \frac{1}{60} \varrho_{\varepsilon}\left(x_{l}\right)\right)\right) .
\end{aligned}
$$

If $y \in U_{0}^{l}$, (14) holds with $x_{\theta}$ for " $x$ ", $F_{\beta}$ for " $F$ ", $\omega_{\psi}$ for " $\omega$ ", $N_{\beta}$ for " $N$ ", and the projection $\pi_{\beta}: E \rightarrow E / F_{\beta}$ instead of " $\pi$ ". The hypotheses of 5.2 are satisfied. From the first inequality of (14) and the definition (15), in which, from (e), one may read $d\left(y, N_{\beta}\right)$ for " $d(y, N)$ ",

$$
\left\|\pi_{\beta} \circ \omega_{\psi}(y)\right\|_{\omega_{\psi}, x_{\theta}}^{\prime \prime}<\frac{1}{200} l^{-1} \varrho_{\varepsilon}\left(x_{l}\right) .
$$

As $\pi_{\beta}$ is linear and $\pi_{\beta} \circ \omega_{\psi}\left(x_{l}\right)=0$, this implies that

$$
\begin{aligned}
\pi_{\beta}\left(A\left(\frac{6}{5}, \omega_{\psi}\left(x_{l}\right)\right) \operatorname{co}\left(\omega_{\psi}\left(U_{0}^{l}\right)\right)\right) & =A\left(\frac{6}{5}, 0\right) \operatorname{co}\left(\pi_{\beta}\left(\omega_{\psi}\left(U_{0}^{l}\right)\right)\right) \\
& =\frac{6}{5} \operatorname{co}\left(\pi_{\beta}\left(\omega_{\psi}\left(U_{0}^{l}\right)\right)\right) \\
& \subseteq B_{\omega_{\psi}, x_{\theta}}^{\prime \prime}\left(0 ; \frac{3}{500} l^{-1} \varrho_{\varepsilon}\left(x_{l}\right)\right),
\end{aligned}
$$

the \|\|$_{\omega_{\psi}, x_{\theta}}^{\prime \prime}$-ball about the origin in $E / F_{\beta}$ of radius $\frac{3}{500} l^{-1} \varrho_{\varepsilon}\left(x_{l}\right)$.

Because of the second inequality of (14), if $y \in B\left(x_{\theta} ; \frac{1}{6} \varrho_{\varepsilon}\left(x_{\theta}\right)\right)$ and $\left\|\pi_{\beta} \circ \omega_{\psi}(y)\right\|_{\omega_{\psi}, x_{\theta}}^{\prime \prime}<\frac{3}{500} l^{-1} \varrho_{\varepsilon}\left(x_{l}\right)$, then

$$
d\left(y, N_{\beta}\right)<\frac{9}{1250} l^{-1} \varrho_{\varepsilon}\left(x_{l}\right)<\frac{1}{120} l^{-1} \varrho_{\varepsilon}\left(x_{l}\right) .
$$

Hence, by (19), (18) and (15), and because $\omega_{\psi}$ is one-one on $B\left(x_{\theta} ; \frac{1}{6} \varrho_{\varepsilon}\left(x_{\theta}\right)\right)$,

$$
\begin{aligned}
& A\left(\frac{6}{5}, \omega_{\psi}\left(x_{l}\right)\right) \operatorname{co}\left(\omega_{\psi}\left(U_{0}^{l}\right)\right) \\
& \quad \subseteq \omega_{\psi}\left(B\left(x_{l} ; \frac{1}{60} \varrho_{\varepsilon}\left(x_{l}\right)\right) \cap\left\{y \in N: d(y, N)<\frac{1}{120} l^{-1} \varrho_{\varepsilon}\left(x_{l}\right)\right\}\right)=\omega_{\psi}\left(U^{l}\right) .
\end{aligned}
$$

Since $\operatorname{co}\left(\omega_{\psi}\left(U_{0}^{l}\right)\right)$ is open, 4.9 shows that $\operatorname{co}\left(\omega_{\psi}\left(U_{0}^{l}\right)\right) \prec \omega_{\psi}\left(U^{l}\right)$. I have now established 3.5 $\left(\mathrm{iv}^{\prime}\right)(\mathrm{b})$ in all cases. Finally, 3.1(iii) follows from (15) and (16).

REMARK 5.4. The above method of proof may be extended, with some additional complications, to show that certain other sets in $M$ are also simply $\mathcal{D}$-extensible: for instance, $\mathcal{D}$-submanifolds with corners and locally finite arrays of $\mathcal{D}$-submanifolds in "general position", as suitably defined. The above case is probably the most interesting. It should be noted that the 
submanifolds need not be split-embedded. Recall, however, that the extension problem concerns functions already defined on a neighbourhood of the supposed core.

The numerical values used in the proof are of course without significance.

The construction satisfies the condition that $\Theta=\left\{j \in J: U^{\theta} \cap N \neq \emptyset\right\}$, which is a natural property to ask of a $\mathfrak{V}$-extension system; it was not, however, required in the extension argument 3.6. If it were imposed, 3.10 could be simplified-see 3.11 .

THEOREM 5.5. Let $\mathcal{C}$ be an admissible category (see 2.2) of mappings between topological vector spaces, and let $\mathcal{C}^{\prime}$ be the subcategory of $\mathcal{C}$ whose objects are normed spaces and whose morphisms are $\mathrm{C}^{1}$ in the sense of Fréchet. Let $M$ be a separable metrizable $\mathcal{L C}^{\prime}$-manifold. Suppose that $X$ is a subset of $M$ that may be expressed as the union of a discrete family of closed $\mathcal{L C}^{\prime}$-submanifolds and of a closed locally compact subset. Then, to any open neighbourhood $U$ of $X$ in $M$, there exists a neighbourhood $V$ of $X$, with $V \subseteq U$, such that

(i) for any locally trivial $\mathcal{L C}$-fibration $\pi: L \rightarrow M$ of $\mathcal{L C}$-manifolds, with fibre $N$ that is $\mathcal{L C}$-diffeomorphic to an open convex set $Y$ in the topological vector space $F$, and to any $\mathcal{L C}$-section $\sigma$ of $\pi$ over $U$, there corresponds an $\mathcal{L C}$-section $\tau$ of $\pi$ over $M$ such that $\sigma|V=\tau| V$; call $\tau$ the "extension" of $\sigma$;

(ii) if $\pi$ is an $\mathcal{L C}$-vector fibration (see 2.9), and a linear space $\mathcal{F}$ of $\mathcal{L C}$-sections of $\pi$ over $U$ is given (with respect to pointwise operations), then the extensions of these sections may be so constructed as to define a linear operator from $\mathcal{F}$ into the space of $\mathcal{L C}$-sections of $\pi$ over $M$;

(iii) for any $\mathcal{L C}$-mapping $g: U \rightarrow F$ into a topological vector space $F$, there is an $\mathcal{L C}$-mapping $f: M \rightarrow F$ (the "extension" of $g$ ) such that $f|V=g| V$ and $f(M) \subseteq \operatorname{co}(g(U))$; furthermore, if a linear space $\mathcal{F}$ of $\mathcal{L}$ mappings $U \rightarrow F$ (with respect to pointwise operations) is given, their extensions may be so constructed as to define a linear operator from $\mathcal{F}$ into the space of $\mathcal{L C}$-mappings $M \rightarrow F$.

As remarked at 3.7, the topology on $F$ is not really important in (iii).

Proof. $X$ is simply $\mathcal{L C}^{\prime}$-extensible by 5.3 and 5.1 ; it follows that it is simply $\mathcal{L C}$-extensible, and the result follows by 3.12 and the remark following it.

Corollary 5.6. Any paracompact $\mathrm{C}^{\infty}$ manifold $M$ modelled on the $B$ nach space $C$ or on the Banach space $\ell^{1}$ admits a non-constant real-valued $\mathrm{C}^{\infty}$ function.

Proof. It suffices to assume $M$ is connected, and therefore separable and metrizable. By [2] $1.3, M$ is an $\mathcal{L} \mathrm{C}^{\infty}$-manifold. For distinct points $a, b \in M$, let $g$ take the values 1 on a neighbourhood of $a$ and 0 on a neighbourhood 
of $b$. By 5.5 , there is an $\mathcal{L} \mathrm{C}^{\infty}$ function $f: M \rightarrow \mathbb{R}$ which agrees with $g$ on some (smaller) neighbourhoods of $a$ and $b$.

6. Other cases. $\S \S 4$ and 5 rely on continuous Fréchet differentiation in normed spaces to satisfy the approximate convexity requirements of 3.5 for a $\mathcal{D}$-manifold. If the model is not normed and the differential calculus employed does not have suitable properties with respect to convex neighbourhoods of the origin (properties that one has no right to expect), arguments such as those must fail. However, 3.6 may still sometimes be used, in a slightly modified version. Instead of introducing an "approximate convex structure" on the manifold by means of a Finsler structure, one may have something given a priori. The simplest example is an open set in a locally convex space, but a little more generality is possible.

TheOREm 6.1. Let $X$ be a subset of a locally convex Hausdorff Lindelöf space $E$, and let $Y$ be a convex subset of a vector space $F$; let $\mathcal{F}$ be a $Y$-admissible class of functions $X \rightarrow Y$ (see [2,1.7]). If $K$ is a subset of $X$ that is closed locally compact in $E$ and $U$ is an open neighbourhood of $K$ in $E$, there is an open neighbourhood $V \subseteq U$ of $K$ in $E$ such that, for any $\mathcal{L F}$-function $g: U \cap X \rightarrow Y$, there is an $\mathcal{L} \mathcal{F}$-function $f: X \rightarrow Y$ for which $f|V \cap X=g| V \cap X$. ( $f$ may be called the "extension" of $g$ ). Moreover, if a whole convex set $\mathcal{E}$ of $\mathcal{L} \mathcal{F}$-functions $U \cap X \rightarrow Y$ (with respect to pointwise operations) is given, their extensions may be so constructed as to define an affine-linear map from $\mathcal{E}$ into the space of $\mathcal{L} \mathcal{F}$-functions $X \rightarrow Y$.

Proof. The details of this proof will be omitted, for it contains no new ideas. As in 5.2, one may construct a $\mathfrak{V}$-extension family for $X$ of the form $\left(\left\{\left(U^{j}, U_{0}^{j}\right): j \in J\right\}, \Theta\right)$, where

$$
U^{j}:=X \cap\left(x_{j}+B_{j}\right), \quad U_{0}^{j}:=X \cap\left(x_{j}+\frac{1}{2} B_{j}\right), \quad \mathfrak{V}:=\{U \cap X, X \backslash K\},
$$

$B_{j}$ an open convex neighbourhood of 0 in $E, \Theta:=\left\{j \in J: U^{j} \cap K \neq \emptyset\right\}$; one takes

$$
V:=\bigcup_{\theta \in \Theta} U_{0}^{\theta} \subseteq \bigcup_{\theta \in \Theta} U^{\theta} \subseteq U
$$

The argument of 3.6 may now be followed, with many simplifications, using $[2,5.1]$ exactly as stated (with the set $X$ ); instead of charts $\omega_{j}$, one has convex subsets of $E$.

\section{References}

[1] C. J. Atkin, Bounded complete Finsler structures I, Studia Math. 62 (1978), 219228.

[2] - Extension of smooth functions in infinite dimensions, I: unions of convex sets, ibid. 146 (2001), 201-226. 
[3] R. A. Bonic and J. Frampton, Smooth functions on Banach manifolds, J. Math. Mech. 15 (1966), 877-898.

[4] R. Deville, G. Godefroy, and V. Zizler, Smoothness and Renormings in Banach Spaces, Pitman Monogr. Surveys Pure Appl. Math. 64, Longman, New York, 1992.

[5] K. D. Elworthy, Embeddings, isotopy and stability of Banach manifolds, Compositio Math. 24 (1972), 175-226.

[6] R. Engelking, General Topology, PWN, Warszawa, 1977.

[7] A. Frölicher and W. Bucher, Calculus in Vector Spaces without Norm, Lecture Notes in Math. 30, Springer, Berlin, 1966.

[8] A. Kriegl and P. W. Michor, The Convenient Setting for Global Analysis, Math. Surveys Monogr. 53, Amer. Math. Soc., Providence, RI, 1997.

[9] S. Lang, Differentiable Manifolds, Wiley-Interscience, New York, 1966.

[10] R. S. Palais, Lusternik-Schnirelman theory on Banach manifolds, Topology 5 (1966), $115-132$.

[11] H. Whitney, Analytic extensions of differentiable functions defined in closed sets, Trans. Amer. Math. Soc. 36 (1934), 63-89.

School of Mathematical and Computing Sciences

Victoria University of Wellington

P.O. Box 600, Wellington, New Zealand

E-mail: chris.atkin@vuw.ac.nz

Received March 13, 2000

Revised version December 27, 2001 